\title{
Raumordnerische Risikovorsorge am Beispiel der Planungsregion Köln
}

\author{
Stefan Greiving ${ }^{1}$ Andrea Hartz $^{2} \cdot$ Florian Hurth $^{3} \cdot$ Sascha Saad $^{2}$
}

Eingegangen: 11. September 2015 / Angenommen: 17. Februar 2016 / Online publiziert: 11. März 2016

(C) Springer-Verlag Berlin Heidelberg 2016

Zusammenfassung Das vorgestellte Modellvorhaben der Raumordnung „Vorsorgendes Risikomanagement in der Regionalplanung" befasste sich mit der exemplarischen Entwicklung, Anwendung und Dokumentation eines integrierten Ansatzes zur Risikovorsorge im Rahmen der Regionalplanung am Beispiel der Planungsregion Köln. Dabei waren die Belange kritischer Infrastrukturen besonders zu würdigen. Die Bearbeitung des Projekts erfolgte im Sinne der Modellprojektforschung des Bundes in enger Kooperation mit den Dezernaten Regionalentwicklung, Wasserwirtschaft und Immissionsschutz der Bezirksregierung Köln. Das Projekt folgte konzeptionell einem integrierten Risiko-Governance Ansatz.

Regionalplanerisch relevant sind dabei Risiken aus solchen Gefährdungen, die im Sinne des $\S 1$ Abs. 1 bzw. $\S 8$ Abs. 6 ROG raumbedeutsam sind: Sie erfordern somit

Prof. Dr.-Ing.Stefan Greiving

stefan.greiving@tu-dortmund.de

Andrea Hartz

andreahartz@agl-online.de

Florian Hurth

hurth@plan-risk-consult.de

Sascha Saad

saschasaad@agl-online.de

1 Institut für Raumplanung, Fakultät Raumplanung, TU Dortmund, August-Schmidt-Str. 10, 44227 Dortmund, Deutschland

2 agl - angewandte geographie, landschafts-, stadt- und raumplanung Hartz - Saad - Wendl, Großherzog-FriedrichStraße 16-18, 66111 Saarbrücken, Deutschland

3 plan + risk consult, Raumplanung und Umweltforschung, Stockumer Str. 435/437, 44227 Dortmund, Deutschland eine überörtliche, überfachliche Betrachtung, weil ihre Auswirkungen bzw. Vermeidungs- und/oder Bewältigungsstrategien von überörtlicher Bedeutung sind. Dieser Definition folgend wurden für die Betrachtung in der Region Köln die vier Gefahrenkomplexe Flusshochwasser, Erdbeben, technische Störfälle und Hitze ausgewählt.

Für diese vier Gefahrenkomplexe wurden Gefährdungsund Empfindlichkeitsstufen in Anlehnung an eine vom Bundesamt für Bevölkerungsschutz und Katastrophenhilfe entwickelte Methodik bestimmt und zu einem Risikoindex verschnitten. Die Einteilung in Gefährdungsstufen wurde in Abstimmung mit den fachlich zuständigen Experten und Expertinnen der Bezirksregierung vorgenommen. Dem gleichen Ansatz folgte die Bestimmung der Empfindlichkeit der einzelnen im Regionalplan festlegbaren Raumnutzungen und Raumfunktionen gegenüber jedem Gefahrenkomplex.

Auf dieser methodischen Grundlage wurde schließlich eine GIS-basierte Risikoanalyse durchgeführt. Die wichtigsten Ergebnisse daraus sind Gefahren-, Empfindlichkeits- sowie Risikokarten für Flusshochwasser, Erdbeben und technische Störfälle - sowohl für die gesamte Region und (mit einer feineren Auflösung) für ausgewählte Hot Spots. Darauf aufbauend wurden Vorschläge für mögliche Plansätze erarbeitet, die sich als Abwägungsgrundlage verstehen. Diese Grundlagen werden von der Bezirksregierung für die anstehende Fortschreibung des Regionalplans genutzt.

Neben den methodischen Bausteinen zur Erarbeitung eines Risikoprofils für Regionen gehört zu den Projektergebnissen zudem ein „Fahrplan“ für ein integriertes Risikomanagement in der Regionalplanung, das die Bezirksregierung Köln bei der Fortschreibung des Regionalplans nutzen wird. Besondere Bedeutung werden diese Erkenntnisse auch für die Umweltprüfung haben, da 
die Behandlung von Klimawandel und die Anpassung an diesen sowie der Umgang mit Katastrophenrisiken durch die Änderungsrichtlinie 2014/52/EU zur gesetzlichen Pflichtaufgabe werden.

Schlüsselwörter Risikomanagement · Regionalplanung · Kritische Infrastruktur · Region Köln

\section{Risk Prevention and Spatial Planning-the Example of the District of Cologne}

\begin{abstract}
With the demonstration project "Preventive Risk Management and Regional Planning" the exemplary development, implementation and documentation of an integrated concept for risk prevention embedded within regional planning was elaborated. The project was based on the concept of risk governance. It was implemented in the case study region of the District of Cologne, which was represented by its departments for regional development, immission control and water management. Within a broader scope of analysis, special attention was paid to critical infrastructures.

For regional planning those risks and hazards are most important that require a cross-sectoral, regional response (see Art. $1 \S 1$ and Art. $8 \S 6$ Federal Regional Planning Act), as their impacts or related response strategies are of regional character. Therefore, the following four hazards were selected: river flooding, earthquakes, major accident hazards and heat.
\end{abstract}

For these four potential dangers hazard and susceptibility classes were defined in accordance with a concept of risk analysis developed by the Federal Office for Civil Protection and Crisis Prevention. Hazard and susceptibility were merged into an integrated risk index. The categorization into hazard classes was the result of a consensus-building process among the project team and the participating experts of the District of Cologne. Furthermore, the team collaboratively defined specific susceptibilities for each land-use function (e. g. residential areas, transport infrastructure etc.) regarding each of the four selected types of hazards.

On this conceptual basis, a GIS-based analysis of the risks that are caused by the selected hazards was conducted. Main results are hazard, susceptibility and risk maps for river floods, earthquakes and major accident hazardsboth for the entire region and (with a finer resolution) for selected hot spots. Moreover, the project developed recommendations for regulations on risk management as a basis for the necessary weighting up of risk prevention against other planning concerns. Finally, a roadmap for spatial risk management as an integrated part of a comprehensive regional plan was derived.
The District of Cologne will use the project's results as an evidence basis for the forthcoming update of the regional plan. This is of particular importance in view of the recent amendment of the EU Environmental Impact Assessment Directive (2014/52/EU) that widened the scope of this assessment to climate change and catastrophic risks.

Keywords Risk management - Regional planning · Critical infrastructure $\cdot$ District of Cologne

\section{Einleitung}

\subsection{Problemstellung}

Risiken betreffen fast alle Bereiche des gesellschaftlichen Lebens. Sie nehmen gerade in Ballungsräumen mit hohen Bevölkerungsdichten und einer Bündelung von (kritischen) Infrastrukturen sowie in Zeiten des Klimawandels zu (Mickwitz/Aix/Beck et al. 2009; UN 2015). Der Monitoringbericht zur Deutschen Anpassungsstrategie an den Klimawandel (UBA 2015) zeigt dessen mögliche Auswirkungen auf zahlreiche Sektoren, wie menschliche Gesundheit, Wasserwirtschaft, biologische Vielfalt, Landund Waldwirtschaft, Siedlung und Verkehr, Finanzwirtschaft oder Bevölkerungsschutz. Darüber hinaus birgt die zunehmende Systemkomplexität gesellschaftlich relevanter technischer Systeme (Computerisierung, Stromabhängigkeit, Informationsabhängigkeit) neue Technikgefahren und -risiken.

Bisher dominiert in der Risikowahrnehmung und -bewältigung in Deutschland eine sektorale Perspektive bzw. Risikomanagement wird eher als fachplanerische Aufgabe wahrgenommen, die primär auf eine Abwehr einzelner Gefahren abzielt. Eine spezifische Rolle der Raumplanung ist hierbei (auch international) eher schwach ausgeprägt (Sapountzaki/Wanczura/Casertano et al. 2011). Erste Ansätze in Deutschland, die das Risiko stärker in der Vordergrund stellen, beziehen sich primär auf den Umgang mit Hochwasser (Seifert 2012; Regionaler Planungsverband Oberes Elbtal/Osterzgebirge 2015), sind aber nicht gängige Praxis in der Regionalplanung - auch nicht beim Umgang mit Hochwasser. Bei anderen Gefahrenkomplexen wie Küstensturmfluten (MKRO 2013) dominiert ebenfalls noch die Gefahrenperspektive bzw. der technische Küstenschutz. Zwar haben sich die verschiedenen Klima-MOROVorhaben unter anderem auch mit Extremwetterereignissen beschäftigt (BMVBS/BBSR 2013a; BMVBS/BBSR 2014) und konnten hier große Fortschritte bei den Anpassungsstrategien an den Klimawandel erzielen. Dabei standen aber nicht raumrelevante Risiken, sondern die Bewältigung von Klimafolgen (und damit auch schleichenden Veränderungen) im Vordergrund, welche einem anderen 
konzeptionellen Grundverständnis folgt (BMVBS/BBSR 2013b; Greiving/Zebisch/Schneiderbauer et al. 2015). So können keine probabilistischen Aussagen etwa zu Hochwasserereignissen in der Zukunft getroffen werden, da die klimawandelbedingten Veränderungen der Frequenz und Magnitude von Extremwetterereignissen mit sehr hohen Unsicherheiten verbunden sind (Field/Barros/Stocker et al. 2012; EEA 2012). Im Übrigen sind nicht alle Risiken (z. B. Erdbeben oder Störfälle) klimawandelgetrieben. Immerhin hat die Ministerkonferenz für Raumordnung (MKRO 2013) in ihrem fortgeschriebenen Handlungskonzept „Raumordnung und Klimawandel" die Notwendigkeit erkannt, eine ergänzende Risikominimierung in sturmflutgeschützten Küstengebieten zu betreiben. Eine gute Grundlage bieten hier die sogenannten „Risikokarten“ gemäß EU-Hochwasserrisikomanagementrichtlinie (HWRM-RL, Richtlinie 2007/60/EG) (EU 2007), die für überflutungsgefährdete Gebiete (auch an den Küsten) vulnerable Landnutzungen darstellen (aber keine Risikokarten im eigentlichen Sinn sind, da nur eine räumliche Überlagerung von Gefährdung und Schutzgut, aber keine Klassifizierung eines Risikos erfolgt).

Mithin wird hier dafür plädiert, Klimawandelanpassung und Risikomanagement - bei allen Überschneidungen - als getrennte Handlungsfelder zu betrachten. Dies ist auch die Sichtweise der Europäischen Kommission, die in der Novelle der Umweltverträglichkeits-Richtlinie beide Themen als neue, aber getrennte Aspekte für die Umweltprüfung ansieht (EU 2014).

Eine vergleichbar einheitliche Evidenzbasis und bundeseinheitliche gesetzliche Grundlage fehlen beispielsweise für alpine Naturgefahren (gravitative Massenbewegungen) völlig (Greiving/Mayer/Pohl et al. 2012). Auch im Kontext technischer Störfälle, die über die Seveso-III-Richtlinie (Richtlinie 2012/18/EU) (EU 2012) bzw. über § 50 Bundesimmissionsschutzgesetz geregelt werden, wird zwar auf angemessene Abstände zwischen Betriebsbereichen und schutzbedürftigen Gebieten geachtet, aber es findet keine (probabilistische) Risikobetrachtung statt, da weder das Wiederkehrintervall der Gefährdung quantifiziert noch die Empfindlichkeit der schutzbedürftigen Gebiete differenziert betrachtet wird (KAS 2010).

Auch eine raumbezogene Multirisikobetrachtung wird in der Praxis der Regionalplanung bisher ausgeblendet, wäre aber angesichts der vielfältigen Wechselwirkungen und kumulativen Effekte etwa zwischen Natur- und Technikgefahren geboten (Greiving 2002; Reckien/Flacke/Dawson et al. 2014). Dies gilt gleichermaßen für das Raumordnungsgesetz (ROG): Es erfolgt keine der überfachlichen Funktion der Raumordnung angemessene raumbezogene Ansprache multipler Risiken. Natürlich lässt sich hier der Grundsatz der Konfliktbewältigung anführen, der sich in der in $\S 1$ Abs. 1 ROG formulierten Aufgabe der Raumordnung wiederfindet:
„(1) Der Gesamtraum der Bundesrepublik Deutschland und seine Teilräume sind durch zusammenfassende, überörtliche und fachübergreifende Raumordnungspläne, durch raumordnerische Zusammenarbeit und durch Abstimmung raumbedeutsamer Planungen und Maßnahmen zu entwickeln, zu ordnen und zu sichern. Dabei sind 1) unterschiedliche Anforderungen an den Raum aufeinander abzustimmen und die auf der jeweiligen Planungsebene auftretenden Konflikte auszugleichen, 2) Vorsorge für einzelne Nutzungen und Funktionen des Raums zu treffen."

Diese Aufgabe besteht zweifelsohne auch im Hinblick auf die Bewältigung raumrelevanter Risiken. Allerdings ist das „Handlungsfeld ,Risikomanagement ${ }^{\text { }}[\ldots]$ in der Raumordnung und Bauleitplanung bislang normativ und planungspraktisch erst in Ansätzen etabliert" (Birkmann/ Greiving/Overbeck et al. 2011: 2). So würdigt das ROG mit $\S 2$ Abs. 2 Nr. 6 Satz 4 zwar einzelne Gefahrenkomplexe und nennt sogar Maßnahmen zur Risikovorsorge: „Für den vorbeugenden Hochwasserschutz an der Küste und im Binnenland ist zu sorgen, im Binnenland vor allem durch Sicherung oder Rückgewinnung von Auen, Rückhalteflächen und Entlastungsflächen." Dagegen werden andere Gefahrenkomplexe wie technische Störfälle oder alpine Naturgefahren überhaupt nicht angesprochen.

Damit fehlt es für die Bewältigung raumrelevanter Risiken in den handlungsleitenden gesetzlichen Grundlagen (ROG, Landesplanungsgesetze und -pläne) an elementaren Voraussetzungen: Was sind raumrelevante Risiken? Wie lassen sich diese analysieren und bewerten? Wie sollen entsprechende Anforderungen abgestimmt und Konflikte zwischen Risikovorsorge und anderen Nutzungen ausgeglichen werden? Dafür sind im Rahmen eines Arbeitskreises der „Akademie für Raumforschung und Landesplanung" " zwar Ansätze entwickelt worden (Greiving/Spangenberg/Zehetmair 2011), die aber bisher nicht in der Planungspraxis verwendet werden.

\subsection{Ziele des Beitrags und methodisches Vorgehen}

Ein vorsorgendes Risikomanagement in der Raumordnung sollte grundsätzlich sektorenübergreifend und ebenenspezifisch entwickelt und in einen strategischen wie dynamischen Ansatz integrierter Raumentwicklung eingebettet werden. Raumordnung verfolgt eine integrierte Perspektive, um die vielfältigen Raumfunktionen und Raumnutzungen im Sinne einer nachhaltigen Raumentwicklung gemäß $\S 1$ ROG in Einklang zu bringen. Ihrem gesetzlichen Auftrag folgend kann sie eine wichtige Rolle in Bezug auf die Risikovorsorge übernehmen. Dies manifestiert sich primär auf der Ebene der Regionalplanung, da die Landesplanung maßstabsbedingt zwar Ziele und Grundsätze formulieren, aber keine differenzierte Risikoanalyse und keine daraus 
abgeleiteten räumlich konkreten Managementansätze entwickeln kann.

Die Intention des Papiers ist es nicht, das Handlungsfeld „Umgang mit Risiken in der Raumplanung“ in Gänze zu beleuchten, sondern einen Beitrag zu einem anwendungsorientierten, in der Praxis auf der Ebene der Regionalplanung umsetzbaren Umgang mit Risiken zu leisten. Insofern steht nicht die theoretische Durchdringung des Themas Risiko, sondern ein für die Planungspraxis adaptiertes Vorgehen im Vordergrund der Betrachtung. Dessen ungeachtet bedarf es der Klärung konzeptioneller und begrifflicher Fragen (vgl. Kapitel 2), um auf einem gemeinsamen Verständnis aufbauend Risiken analysieren und Risikovorsorge betreiben zu können. Der Handlungsauftrag von Raumordnung im Risikokontext ist Gegenstand von Kapitel 3, während sich Kapitel 4 der Risikoanalyse widmet, die als Evidenzbasis für eine raumordnerische Risikovorsorge dient. Für diese werden Handlungsansätze vorgestellt und mit der Planungspraxis abgestimmt (Kapitel 5). In Kapitel 6 wird dazu schließlich ein Fazit gezogen, das die eingangs gestellten Zielsetzungen kritisch reflektiert.

Der Beitrag stützt sich auf die Ergebnisse des Modellvorhabens (MORO) „Vorsorgendes Risikomanagement in der Regionalplanung“ (BMVI/BBSR 2015). ${ }^{1}$ Der methodische Ansatz wurde am Beispiel einer Planungsregion konkretisiert, um praxistaugliche Empfehlungen aus der Erprobung auch für andere Planungsregionen abzuleiten. Forschung im Rahmen von Modellvorhaben zählt zu den Ansätzen diskursiver Politikberatung: Experten und politische Akteure erzeugen gemeinsam im Prozess ihrer Interaktion Verfahrens- oder Instrumenteninnovationen. Durch die Berücksichtigung der Erfahrungen der beteiligten Praxisakteure erzielt Modellvorhabenforschung in der Regel bessere „Lernerfolge“ und ist effektiver als klassische Politikberatung oder rein wissenschaftliche Forschung (Einig 2011: 441).

Als Modellregion wurde der Regierungsbezirk Köln ausgewählt. Entscheidend hierbei ist die Zuständigkeit der Bezirksregierung als Bündelungsbehörde für die Regionalplanung sowie die raumrelevanten Fachplanungen, die durch die Dezernate Regionalentwicklung, Immissionsschutz und Wasserwirtschaft vertreten waren. Zudem steht die Gesamtfortschreibung des Regionalplans bevor, sodass das Modell-

\footnotetext{
${ }^{1}$ Das Modellvorhaben „Vorsorgendes Risikomanagement in der Regionalplanung" wurde vom Bundesministerium für Verkehr und digitale Infrastruktur (BMVI) in Kooperation mit dem Bundesinstitut für Bau-, Stadt- und Raumforschung (BBSR) unter dem Aktenzeichen 10.05.06-13.6 von November 2013 bis Juli 2015 gefördert. Auftragnehmer waren die Büros agl - angewandte geographie, landschafts-, stadt- und raumplanung Hartz - Saad - Wendl, Sarbrücken, und plan + risk consult - Prof. Dr. Greiving \& Partner - Ingenieurgesellschaft für Raumplanung und Umweltforschung, Dortmund.
}

vorhaben zeitgleich mit den ersten Vorbereitungen für den zukünftigen Regionalplan stattfand.

Die Aufgaben der Begleitforschung bestanden darin, zusammen mit der Bezirksregierung Köln.

- die Raumrelevanz von Risiken festzustellen,

- Leitvorstellungen für den planerischen Umgang mit Risiken zu diskutieren,

- ein regionales Risikoprofil im Sinne einer Risikoabschätzung auf der Basis der Ermittlung von Gefahrenquellen und Verwundbarkeiten zu erarbeiten,

- die methodischen Ansätze der Risikoanalyse zu erproben und weiterzuentwickeln,

- den Umgang mit kritischen Infrastrukturen in der Regionalplanung zu erörtern,

- Zuständigkeiten für Risikoanalyse, -bewertung und -bewältigung (innerhalb und außerhalb der Raumplanungsinstitutionen) zu klären,

- Anforderungen an die Risikokommunikation im Kontext der räumlichen Risikovorsorge zu thematisieren sowie regionalplanerische Maßnahmen der Risikovorsorge zu identifizieren.

\section{Verständnis zentraler Begriffe}

Im Folgenden werden die zentralen Begriffe definiert, die für die gemeinsam mit der Bezirksregierung Köln durchgeführte Risikoanalyse maßgeblich waren (sie selbst wird in Kapitel 4 näher vorgestellt). Dabei sind sich die Autoren darüber im Klaren, dass jeder dieser Begriffe Gegenstand umfänglicher wissenschaftlicher Diskussionen ist und alles andere als Konsens, etwa zwischen Natur- und Sozialwissenschaften, über deren Verwendung besteht (vgl. für andere WBGU 1999). Diese Kontroversen zu diskutieren oder gar aufzulösen, ist aber nicht Gegenstand dieses Beitrags.

Das im Projekt verwendete und mit der Bezirksregierung abgestimmte Risikokonzept folgt dabei als Grundlage für die in Kapitel 4 vorgestellte Risikoanalyse zunächst im Grundsatz dem naturwissenschaftlichen Risikoverständnis. Demnach liegt einem (raumrelevanten) Risiko, das sich räumlich manifestiert, stets eine Art von realer Gefahr zugrunde: Gefahr wird als der Tatbestand einer objektiven Bedrohung durch ein zukünftiges Ereignis definiert, wobei die Gefährdung („hazard“) mit einer bestimmten Eintrittswahrscheinlichkeit auftritt. Zum Risiko wird eine Gefahr durch die zu erwartenden Schäden bzw. Folgen des Ereignisses, die durch den Begriff „Verwundbarkeit“ beschrieben werden. Verwundbarkeit setzt sich wiederum zusammen aus der Exposition von Schutzgütern gegenüber Gefährdungen und ihrer Empfindlichkeit den Wirkungen dieser Gefährdungen gegenüber (vgl. Abbildung 1). 


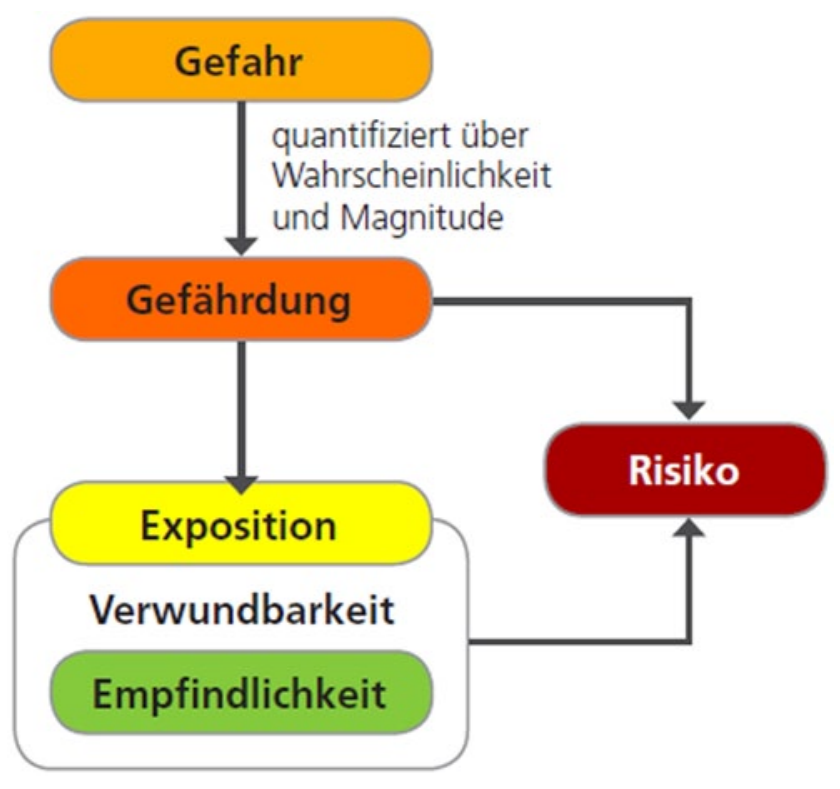

Abb. 1 Risikokonzept (eigene Darstellung in Anlehnung an UN 2009; in BMVI/BBSR 2015: 12)

Risikoanalyse und Risikomanagement in der Regionalplanung sind Bestandteil formeller Planungsprozesse, in deren Kontext die Akteursbeteiligung eine große Rolle spielt. In diesem Zusammenhang ist das sogenannte „Risiko-Governance-Konzept“ zu erwähnen (Renn 2008). Eine Entscheidungsfindung, welche sich lediglich an einer auf naturwissenschaftlichem Risikoverständnis fußenden und durch Experten ermittelten Risikoanalyse orientiert und dabei „sozial-kulturelle“ lebensweltliche Dimensionen nicht berücksichtigt, führt zu Misstrauen (Löfstedt 2005). Ein anderes Entscheidungsproblem besteht darin, dass messbare Fakten angesichts bestehender Unterschiede in der individuell und sozial geprägten Risikowahrnehmung unterschiedlich gewertet werden können (Klinke/ Renn 2002). Dies stellt nicht das naturwissenschaftliche Risikoverständnis in Frage, das bei der in Kapitel 4 vorgestellten Risikoanalyse verwendet wird. Es erfordert aber, dass die naturwissenschaftliche Expertise um eine aktive Akteursbeteiligung ergänzt wird, die im Übrigen auch die EU-Hochwasserrisikomanagementrichtlinie mit Artikel 10 vorsieht. Gegenüber dem klassischen Umgang mit Risiken, bei dem die Gefahrenabwehr dominiert und innerhalb dessen die Akteure allenfalls über die Ergebnisse eines Verwaltungsverfahrens informiert werden (Greiving 2005), bedeutet dies insofern einen Paradigmenwechsel. Risikokommunikation zwischen Wissenschaft und Verwaltung und zwischen Verwaltung und Öffentlichkeit sollte während des gesamten Prozesses stattfinden und daher bereits während der Problemdefinition und Durchführung der Risikoanalyse beginnen. Das Einrichten einer RisikoGovernance-Struktur, das Herstellen von Akteursbezügen sowie das Einbinden relevanter Akteure in den Governance-
Prozess stellen Herausforderungen für die Regionalplanung dar, da es sich bei der Risikovorsorge nicht um ein etabliertes Themenfeld handelt (Glade/Greiving 2011). Zudem findet der Risiko-Governance-Ansatz insbesondere im Kontext der Bewältigung von Naturgefahren bislang kaum Berücksichtigung (Walker/Tweed/Whittle 2014).

\section{Handlungsauftrag der Raumordnung}

\subsection{Raumplanungsrelevanz von Risiken}

Der Risikobegriff ist kein traditioneller Rechtsbegriff vergleichbar dem Gefahrenbegriff: Im öffentlichen Ordnungsrecht zielt das technische Sicherheitsrecht primär auf die Gefahrenabwehr (Greiving 2005). Gefahr bezeichnet nach dem immer noch gültigen Verständnis des Preußischen Oberverwaltungsgerichts eine Lage, in der bei ungehindertem Ablauf des Geschehens ein Zustand oder ein Verhalten mit hinreichender Wahrscheinlichkeit zu einem Schaden durch äußere Einflüsse führen würde (Davy 1990: 306).

Nun dürfte es unstrittig sein, dass Risiken zu Raumnutzungskonflikten führen können. Dies kann der Fall sein, wenn beispielsweise eine Raumfunktion bzw. -nutzung (z. B. Wasserrückhaltung oder großtechnische Anlagen) als Gefahrenquelle für andere Raumnutzungen gilt (z. B. Siedlungsbereiche), sofern letztere gegenüber dieser Gefahrenquelle exponiert sind, das heißt, sie sich in ihrem Einwirkungsbereich befinden und empfindlich gegenüber diesen Einwirkungen (z. B. Überschwemmungen) sind. Zugleich lässt sich die in $\S 1$ Abs. 1 ROG formulierte Maßgabe, Vorsorge für Raumfunktionen und Raumnutzungen zu treffen, als Handlungsauftrag zur Risikovorsorge begreifen.

Aus der Perspektive der Raumordnung zielen mögliche Handlungsoptionen in der Risikovorsorge deshalb.

- auf den Bestand und die Planung von Raumnutzungen und kritischen Infrastrukturen, etwa durch Information und Kommunikation über gebietsbezogene Risiken: Liegt die Einrichtung/Organisation in einem gefährdeten Gebiet? Wie stark ist die Gefährdung (Frequenz und Magnitude) unter Berücksichtigung der für diese Infrastrukturen typischen Anlagensicherheit?

- auf vorbeugende Maßnahmen: flächen- und gebietsbezogene Schutzanforderungen gegenüber Gefährdungen für definierte Schutzgüter, das heißt Raumnutzungen oder -funktionen, die gegenüber den Gefährdungen verwundbar sind. Dies schließt kritische Infrastrukturen wie auch Gefährdungen mit ein, die von diesen im Versagensfall ausgehen.

Regionalplanerisch relevant sind dabei Risiken, die im Sinne des $\S 1$ Abs. 1 bzw. $\S 8$ Abs. 6 ROG raumbedeut- 
sam sind (also eine überörtliche, überfachliche Betrachtung erfordern, weil ihre Auswirkungen bzw. die entsprechenden Vermeidungs- und/oder Bewältigungsstrategien von überörtlicher Bedeutung sind). Dies bedeutet nach $\S 3$ Abs. 6 ROG, dass Raum in Anspruch genommen wird oder die räumliche Entwicklung oder Funktion eines Gebiets durch Risiken beeinflusst wird, einschließlich des Einsatzes der hierfür vorgesehenen öffentlichen Finanzmittel. Maßgeblich sind hier die Möglichkeiten der Beeinflussung der Eintrittswahrscheinlichkeit (Gefährdung infolge Frequenz und Magnitude) sowie des Schadensausmaßes (abhängig von der Verwundbarkeit) durch regionalplanerische Maßnahmen.

Ein Risiko ist also dann raumplanungsrelevant, wenn mithilfe raumplanerischer Instrumente die Eintrittswahrscheinlichkeit oder Konsequenz eines Ereignisses für bestimmte, hinlänglich sicher identifizierbare Entstehungsund/oder Gefährdungsräume beeinflussbar sind. Der Umgang mit Störfällen ist dabei ein spezifischer Fall, da er nur in denjenigen Bundesländern raumordnungsrelevant ist, die auf der Ebene der Regionalplanung über eine positivallokative Standortsteuerung verfügen. Störfälle (bzw. die sogenannten „Achtungsabstände“ zu Störfallbetrieben) sind hingegen in den anderen Bundesländern ausschließlich im Rahmen der Bauleitplanung zu behandeln. Demgegenüber sind virtuelle Risiken (wie etwa die Finanzmarktkrise) oder ubiquitär auftretende Ereignisse (wie etwa eine Pandemie) nicht Gegenstand raumplanerischen Risikomanagements.

Selbstverständlich bedürfen Risiken in vielen Fällen sowohl einer raumordnerischen als auch einer bauleitplanerischen Bewältigung, wie das Beispiel Hochwasser verdeutlicht: Die überörtlich und überfachlich abzuwägende Festlegung von Vorrang- und Vorbehaltsgebieten obliegt der Raumordnung; der verbleibende Regelungsbedarf bzw. der bodennutzungsbezogene Teil fällt in die Verantwortung der Bauleitplanung (Greiving 2011: 24).

\subsection{Bedeutung kritischer Infrastrukturen}

Im Zusammenhang mit hoher Vulnerabilität sind auch kritische Infrastrukturen (KRITIS) zu sehen, die bewusst Gegenstand der Risikoanalyse sein sollten: Kritische Infrastrukturen (Organisationen oder Einrichtungen) besitzen eine zentrale Bedeutung für das staatliche Gemeinwesen und daher eine besondere Schutzwürdigkeit, die unabhängig von ihrer Empfindlichkeit besteht (Riegel 2015). Wie diese normative Komponente angemessen in einer Risikoanalyse zu berücksichtigen ist, ist eine bis dato völlig offene Frage, zumindest in Deutschland. Dabei kann ein Ausfall oder eine wesentliche Störung einer kritischen Infrastruktur zu erheblichen nachteiligen Folgen für das gesellschaftliche Funktionieren führen (BMI 2009; BBK 2009; Krings 2010). Das Bundesministerium des Inneren spricht von zwei relevanten sogenannten „Schadensbildern“ (BMI 2009: 13):
- Schadensbild 1: Weiträumige Einwirkungen auf kritische Infrastrukturen, die insbesondere von Naturgefahren ausgelöst wurden (Flusshochwasser, Winterstürme etc.).

- Schadensbild 2: Lokale Störungen oder Schäden führen $\mathrm{zu}$ Beeinträchtigungen, die vereinzelt weit über das ursprüngliche Schadensgebiet hinauswachsen (z. B. langfristige Sperrung der ICE-Strecke Köln-Berlin bei Fischbeck von Juni bis November 2013). Eine besondere Herausforderung für die Risikoanalyse ist dabei die Erfassung der systemischen Dimension kritischer Infrastrukturen, die über ihre rein baulich-räumliche Komponente, also den Standort bzw. die Trasse der Infrastruktur, hinausgeht.

\subsection{Rolle der Umweltprüfung}

Für die Analyse von Risiken in der Raumordnung bedarf es geeigneter Trägerverfahren. Ein solches stellt prinzipiell die Umweltprüfung dar (Greiving 2004), wenngleich diese in der Praxis bislang nicht hinreichend gewürdigt wird. Insbesondere beim Blick in die Anhänge I und II der SUPRichtlinie (EU-Richtlinie über die strategische Umweltprüfung) (EU 2001) fällt auf, dass hinsichtlich der Kriterien für die Bestimmung der voraussichtlichen Erheblichkeit von Umweltauswirkungen im Sinne des Artikels 3 Abs. 5 eine Reihe von Merkmalen der Auswirkungen und der voraussichtlich betroffenen Gebiete genannt werden, die einen inhaltlichen Bezug zum Risikobegriff aufweisen:

- Wahrscheinlichkeit, Dauer, Häufigkeit und Umkehrbarkeit der Auswirkungen,

- der kumulative Charakter der Auswirkungen,

- die Risiken für die menschliche Gesundheit oder die Umwelt (z. B. bei Unfällen),

- der Umfang und die räumliche Ausdehnung der Auswirkungen (geographisches Gebiet),

- die Anzahl der voraussichtlich betroffenen Personen,

- die Bedeutung und die Sensibilität des voraussichtlich betroffenen Gebiets.

Daran wird unmittelbar deutlich, dass ein Risikobegriff der SUP-Richtlinie nicht nur immanent ist, sondern dass der ganze Ansatz der Richtlinie (,Vorhaben, von denen erhebliche Beeinträchtigungen ausgehen können“) auf mögliche Folgen im Sinne von Risiken abstellt.

Die planerische Herausforderung besteht dabei darin, für jedes Schutzgut plausible Schutzziele festzulegen, die sowohl die bestehende Gefährdung aufgrund möglicher Natur- oder Technikereignisse berücksichtigen als auch die unterschiedliche Schutzwürdigkeit der einzelnen Schutzgüter und ihre spezifische Empfindlichkeit gegenüber unterschiedlichen Ereignissen beachten. Kritische Infrastrukturen sind hier seitens des Gesetzgebers als besonders schutzwürdig erkannt worden (§ 2 Abs. 2 Nr. 3 Satz 4 ROG). 
Die UVP-Änderungsrichtlinie, die im August 2014 in Kraft getreten ist (Richtlinie 2014/52/EU) (EU 2014), hat Artikel 3 um einen Absatz 2 ergänzt, wonach im Rahmen der Umweltverträglichkeitsprüfung (UVP) berücksichtigt werden muss, welche Umweltauswirkungen von einem Projekt im Falle eines schweren Unfalls oder einer Katastrophe zu erwarten sind. Damit wird die Durchführung einer Risikoabschätzung zur gesetzlichen Pflichtaufgabe innerhalb der Umweltprüfung, da diese aufgrund der sog. „Integrationslösung“" sowohl den Anforderungen der UVP als auch der SUP-Richtlinie Genüge tun muss (Battis/ Moench/Uechtritz et al. 2015: 6). Diese Novelle erweitert die bisherige Sichtweise der Umweltprüfung. Dabei kann ein Vorhaben, aber auch ein Projekt oder Plan sowohl die Gefährdung gegenüber einem Natur- oder Technikereignis erhöhen, indem etwa über Bodenversiegelungen der Abfluss beschleunigt oder durch Abholzung ein Hang destabilisiert wird, als auch die Empfindlichkeit erhöhen, indem etwa zusätzliche Schadenspotenziale in von Extremereignissen gefährdeten Bereichen zugelassen werden.

\subsection{Fahrplan für ein integriertes Risikomanagement}

Unabhängig von ihrer institutionellen Verfasstheit kann die Regionalplanung aber keine insgesamt koordinierende Rolle im integrierten Risikomanagement wahrnehmen, sofern Risikomanagement im Sinne des sogenannten „Risikokreislaufs“ aus Vorbereitung, Reaktion, Wiederaufbau und Vorsorge verstanden wird (Pohl 2011). Die Rolle der Regionalplanung bezieht sich vielmehr primär auf die (planerische) räumliche Risikovorsorge. Zu diesem Zweck sind Risiken zu analysieren und entsprechend ihrem Gewicht in die planerische Abwägung einzustellen.

Sowohl die Fachplanungen als auch die Raumplanung agieren räumlich. Dabei nehmen die Fachplanungen meist keine gesamträumliche Perspektive ein. Darüber hinaus variiert das Verhältnis von Fachplanungen zur Raumplanung in den einzelnen Ländern. Ziel sollte es deshalb sein, dass die Raumordnung ihren integrativen Koordinationsanspruch beibehält. Allerdings bedarf dies bei der räumlichen Risikovorsorge in besonderem Maße einer engen Abstimmung mit den Fachplanungen - hinsichtlich der Erarbeitung von Risikoprofilen, der Umsetzung von Strategien zur Risikovorsorge wie auch hinsichtlich einer effektiven Verknüpfung mit dem Katastrophenschutz und dem Wiederaufbau im Katastrophenfall.

Für die kommunale Ebene soll die Raumplanung transparent vermitteln, welche risikorelevanten Informationen und Sachverhalte sowie welche überregionalen Zusammenhänge bedeutsam sind. Die kommunale Ebene verfügt meist über genauere und kleinräumige Informationen und sollte auch im Hinblick auf die überregionalen Belange der Risikovorsorge mehr Gewicht verleihen. Kommunal ver- fasste Regionen wie in Nordrhein-Westfalen können dabei die kommunalen Interessen bei den Themenstellungen des Regionalplans stark beeinflussen.

Der „Fahrplan“ für ein integriertes Risikomanagement in der Regionalplanung als ein wesentliches Ergebnis des abgeschlossenen MORO (BMVI/BBSR 2015: 139) zeigt idealtypisch die zentralen Aspekte der räumlichen Risikovorsorge und deren Interdependenzen auf (Abbildung 2).

\section{Risikoanalyse und Risikobewertung}

\subsection{Grundansatz der Risikoanalyse}

Für einen sachgerechten Umgang mit Risiken ist eine entsprechende Evidenzbasis erforderlich: Hierzu zählen in erster Linie Risikoanalysen und -bewertungen und deren Auswertung in Bezug auf zu bewältigende Raumnutzungskonflikte mit anderen Raumnutzungen und Raumfunktionen. Daraus können Erfordernisse zur Risikobewältigung abgeleitet werden.

Wie misst man nun die Risikobelastung eines Raums? Hier wird vorgeschlagen, der Methodik des Bundesamts für Bevölkerungsschutz zur Risikoeinstufung (BBK 2010: 22) zu folgen (Abbildung 3).

Das Risiko beschreibt in dieser Grundlogik in der Regel eine Funktion aus der Eintrittswahrscheinlichkeit einer Gefahr und dem Schadensausmaß. Die Risikomatrix wird individuell für jeden einzelnen Gefahrenkomplex angepasst. Für den regionalplanerischen Kontext wird als Indikator für die Eintrittswahrscheinlichkeit einer Gefahr (sowie für die Wahrscheinlichkeit negativer Folgen einer Handlung/Entscheidung) gegebenenfalls auch die Magnitude der Gefährdung berücksichtigt, z. B. die Wassertiefe und Fließgeschwindigkeit bei einer Überschwemmung durch Flusshochwasser. Für das Schadensausmaß wird die Verwundbarkeit, bestehend aus Exposition und Empfindlichkeit, als Indikator herangezogen. Die besondere Schutzwürdigkeit, die bestimmten Raumnutzungen (wie etwa kritischer Infrastruktur) unabhängig von ihrer Empfindlichkeit gegenüber bestimmten Gefährdungen zuzubilligen ist, ist nicht innerhalb der Risikoanalyse bestimmbar, sondern fließt in den planerischen Entscheidungsvorgang ein und ist damit als Teil der Risikobewertung (Ist ein ermitteltes Risiko akzeptabel?) anzusehen.

Die Risikomatrix bildet die Verknüpfungsregel zur Bestimmung der Größe eines Risikos. Sowohl die Gefährdung als auch die Verwundbarkeit werden in Gefährdungs- bzw. Empfindlichkeitsstufen (z. B. hoch, mittel, gering) differenziert. Die Gefährdung basiert dabei soweit möglich auf Fachnormen: Für die Arbeiten im Regierungsbezirk Köln wurde für Flusshochwasser die Hochwasserrisikomanagementrichtlinie herangezogen, für 
Abb. 2 „Fahrplan“ für ein integriertes Risikomanagement im Sinne der räumlichen Risikovorsorge in der Regionalplanung (eigene Darstellung; in Teilen angelehnt an Zehetmair 2011: 65; in: BMVI/BBSR 2015: 139)

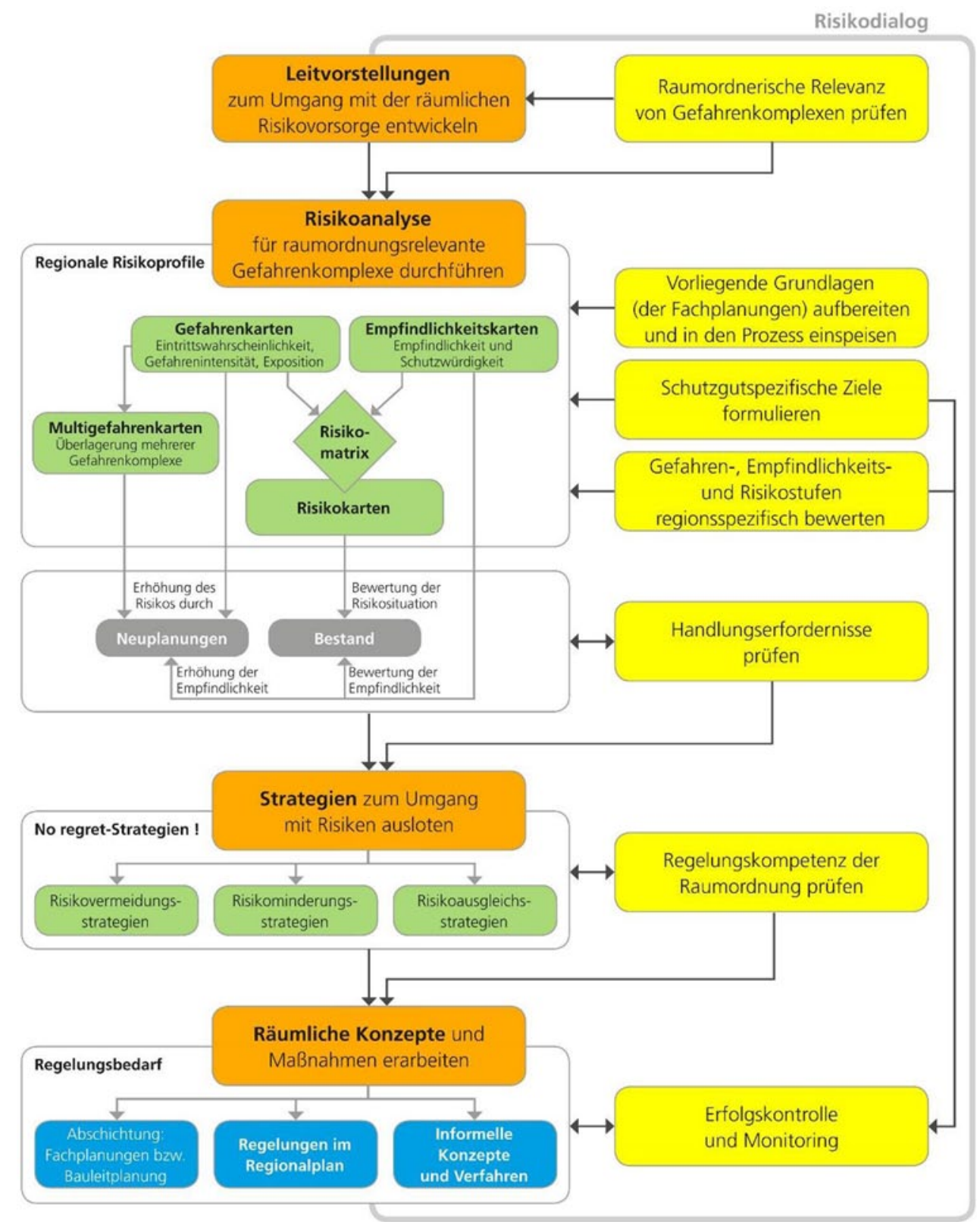

technische Störfälle die Seveso-III-Richtlinie, für Erdbeben die DIN 4149. Lediglich für den Gefahrenkomplex Hitze fehlen bisher Fachnormen.

Da „nicht exponierte Flächen und Objekte“ (keine Gefährdung) in der Risikomatrix nicht dargestellt werden, kann anstelle der Verwundbarkeit unmittelbar die „Bedeutung“ bzw. „Empfindlichkeit“ als Indikator herangezogen werden, um das Risiko zu bestimmen.

\subsection{Risikoanalyse im Planungsraum Köln}

Ein wesentlicher Baustein war die gemeinsame Erarbeitung eines Risikoprofils im Kontext der Erfordernisse der Regionalplanung: Auf der Grundlage der Risikomatrix für die einzelnen Gefahrenkomplexe wurden die zugehörigen räumlichen Informationen zu Gefährdung und Empfindlichkeit zum Risiko im Geographischen Informationssystem (GIS) verschnitten. Die kartographischen Darstellungen der Risikobewertung einzelner Gefahrenkomplexe zeigen daher in drei separaten Karten die Gefährdung, die Empfindlichkeit sowie die Gebiete unterschiedlich großer Risiken auf. Ein entsprechendes Vorgehen könnte zukünftig innerhalb der Umweltprüfung verankert werden und mithin einen Teil des Umweltberichts darstellen. Das Ergebnis der Risikoanalyse geht dann in die planerische Abwägung ein und sollte für den Fall, dass Risiken als akzeptabel angesehen werden, in entsprechende raumordnerische Festlegungen münden (vgl. Kapitel 5).

Die folgenden Abbildungen 4, 5 und 6 verdeutlichen dieses Vorgehen um der Lesbarkeit willen anhand von jeweils 


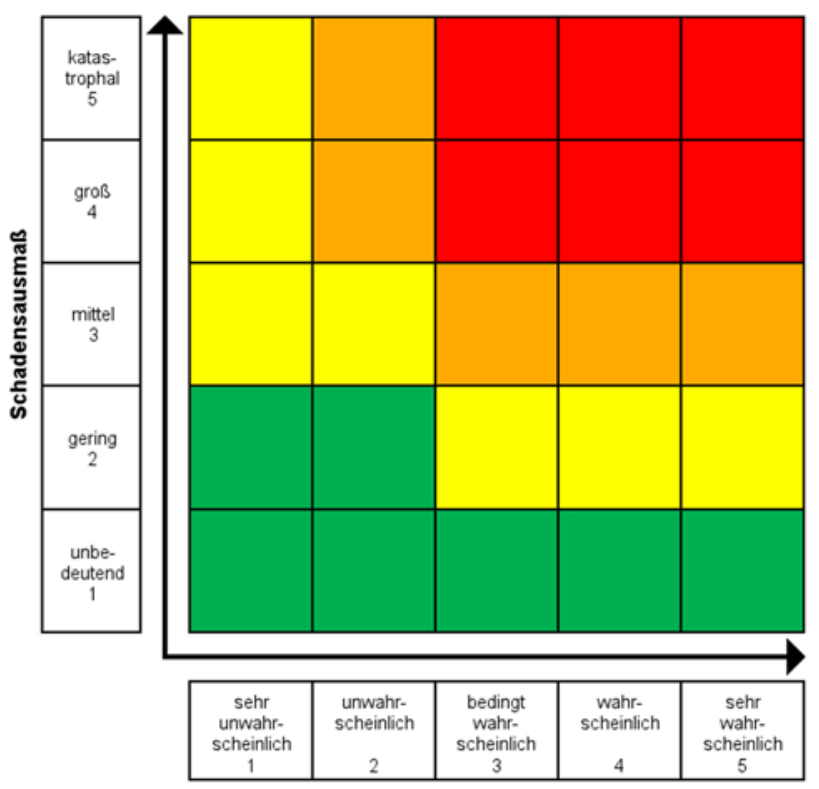

Eintrittswahrscheinlichkeit

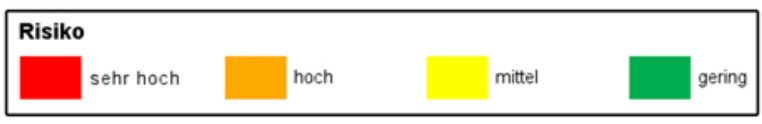

Abb. 3 Risikomatrix (BBK 2010: 22)

zwei kommunalen Beispielen (Leverkusen, Linnich); die Karten wurden aber für den gesamten Planungsraum erstellt. $^{2}$

Die Karten können in der Regionalplanung vielfältig eingesetzt werden:

- Mithilfe der (schutzgutbezogenen) Gefahrenkarten in Verbindung mit den Empfindlichkeitseinstufungen können insbesondere Neuplanungen aus der Perspektive der Risikovorsorge eingeschätzt werden: Sie ermöglichen eine Aussage darüber, inwieweit neue Raumnutzungen bzw. -funktionen zu einer Erhöhung der Risiken im Raum führen können.

- Die Empfindlichkeitskarten geben einen Überblick über bestehende empfindliche Raumnutzungen und -funktionen gegenüber einem Gefahrenkomplex. Zudem ermöglichen sie Aussagen zu einer Erhöhung der Empfindlichkeiten durch Neuplanungen in einem Raum.

- Die Risikokarten erlauben eine Bewertung der Bestandssituation sowie des Handlungsbedarfs, der sich aus dem aktuellen Risikoprofil ergibt.

- Multigefahrenkarten wurden für den Regierungsbezirk Köln als übereinander liegende Gefahrenstufen von Erdbeben, Hochwasser und technischen Störfällen erstellt. Diese können vor allem dazu verwendet werden, Multigefahren aus Schutzgutperspektive zu betrachten.

\footnotetext{
${ }^{2}$ Diese Karten basieren auf Erhebungen der Autoren im Rahmen des MORO und nicht dem Siedlungsflächenmonitoring der Regionalplanungsbehörde Köln.
}

Die Frage, welche Risiken betrachtet bzw. welche Indikatoren für Risiken zugrunde gelegt werden sollen, ist weder aus fachlicher noch aus politischer Perspektive abschließend bestimmt. Dies gilt ebenso für die Abstufung der unterschiedlichen Gefährdungsklassen bzw. Verwundbarkeits-/ Empfindlichkeitsklassen innerhalb einer Risikomatrix sowie die darauf aufbauende Risikobewertung, innerhalb derer auch die Frage nach einer spezifischen Schutzwürdigkeit bestimmter Raumnutzungen zu beantworten ist. Dieses sind im Kern normative Fragen (Slovic 1999; Löfstedt 2005). Im Rahmen des Modellprojekts wurden deshalb die Indikatoren für Gefährdung und Verwundbarkeit sowie deren Abstufungen zur Bestimmung der unterschiedlichen Risiken im Sinne der Modellprojektforschung über eine Befragung der beteiligten regionalen Akteure festgelegt.

\subsection{Umgang mit Unsicherheit in Risikoanalyse und Risikobewertung}

In der Risikoanalyse kann in aller Regel nicht sicher bestimmt werden, welches Ereignis wann und wo auftritt. Damit sind Entscheidungen über den Umgang mit Risiken stets Entscheidungen unter Unsicherheit, die aus verschiedenen Quellen erwächst:

- Die verwendeten Modelle bilden unter Umständen die Realität nur unzureichend ab. Dies ist insbesondere im Bereich Klimafolgenforschung immer noch der Fall. Gerade bei Extremereignissen wie dem 500-jährlichen Hochwasser oder dem 475-jährlichen Erdbeben als Bemessungsfall für die DIN 4149 liegen keine ausreichend langen Beobachtungsperioden vor, um deren Wahrscheinlichkeit bzw. Magnituden präzise einschätzen zu können. Immerhin konnte im Hochwasserbereich durch die Verwendung von 2D-Modellierungen die modellinhärente Unsicherheit reduziert werden.

- Für Störfälle ist selbst bei gutachterlicher Berechnung der Freisetzung von Stoffen und deren Ausbreitung die Quelle von Unsicherheit darin zu sehen, dass keine Wahrscheinlichkeiten für den angenommenen „Dennoch-Störfall“ ${ }^{\star 3}$ bestimmbar sind. Zudem liegen kaum Erfahrungen zum Ablauf tatsächlicher Unfälle vor.

- Es gibt keine etablierten Verfahren zur Beurteilung von Empfindlichkeit und Schutzwürdigkeit der verschiedenen Raumfunktionen und Raumnutzungen.

Entscheidungen unter Unsicherheit sind grundsätzlich in das Konzept der planerischen Entscheidung einzuordnen

\footnotetext{
${ }^{3}$ Dennoch-Störfälle stellen eine „Ausweitung von Betriebsstörungen dar, die trotz störfallverhindernder Maßnahmen, aber aufgrund des Wirksamwerdens einer vernünftigerweise auszuschließenden Gefahrenquelle oder des zeitgleichen Wirksamwerdens mehrerer voneinander unabhängiger Gefahrenquellen" eine ernste Gefahr hervorrufen (KAS 1999: 6).
} 
Abb. 4 Schutzgutbezogene Gefahrenkarten Hochwasser (eigene Darstellung; in BMVI/ BBSR 2015: 98). ASB Allgemeinen Siedlungsbereiche, GIB Bereiche für gewerbliche und industrielle Nutzungen

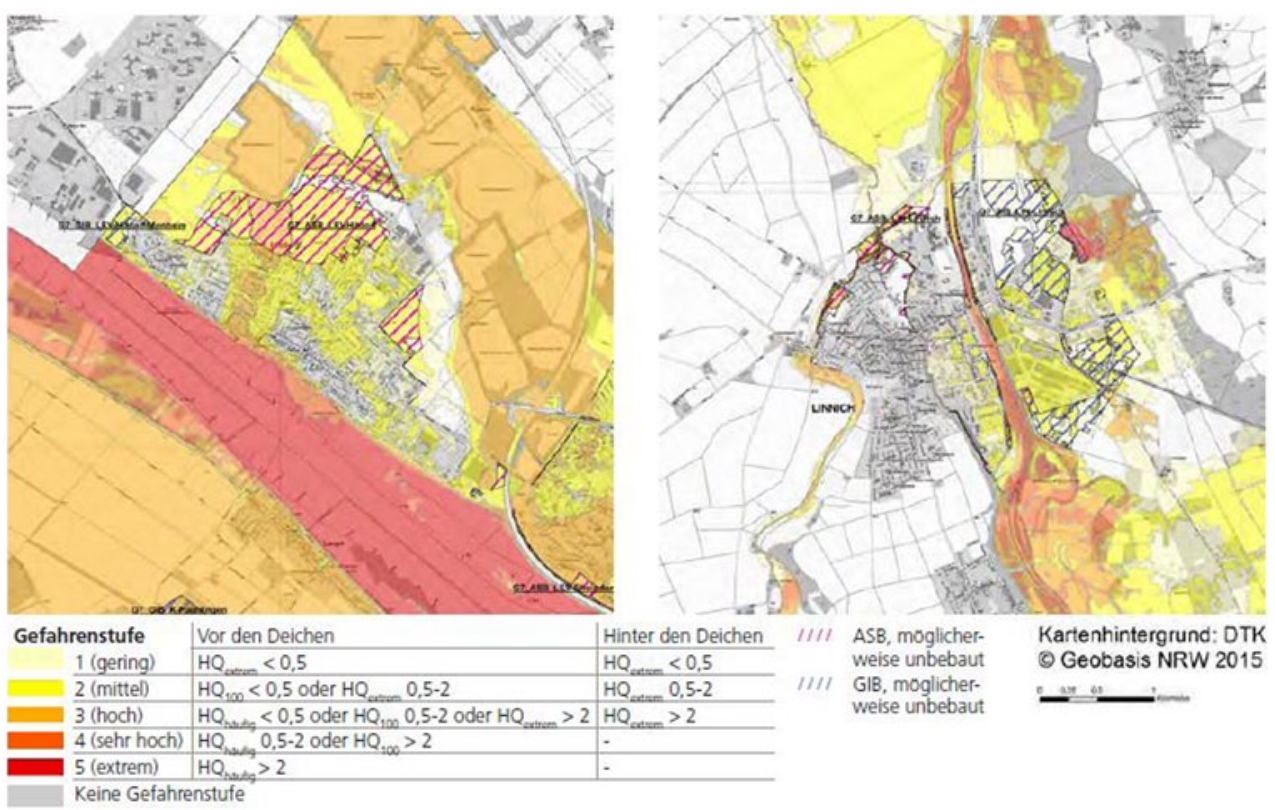

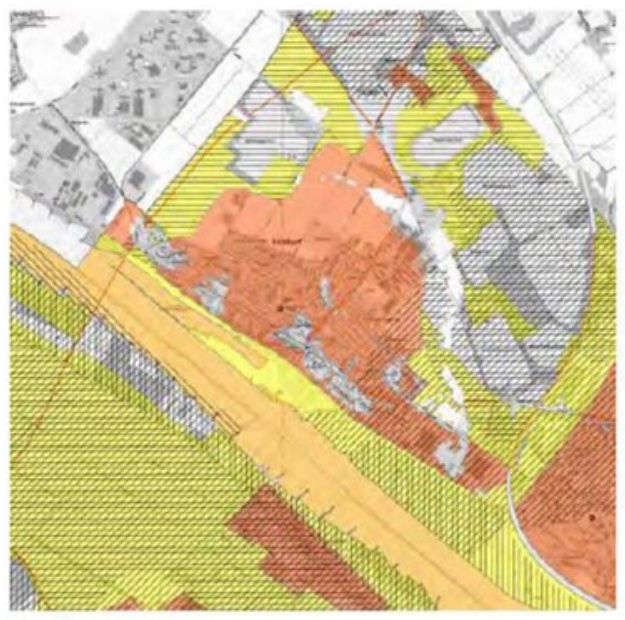

Empfindlichkeitsstufe

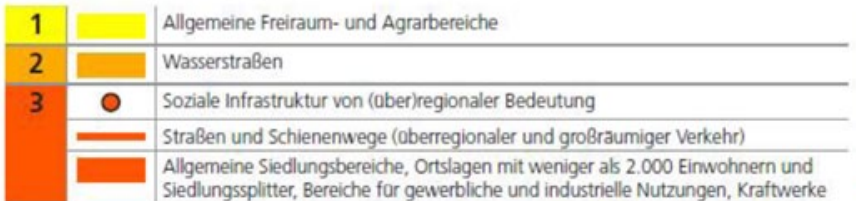

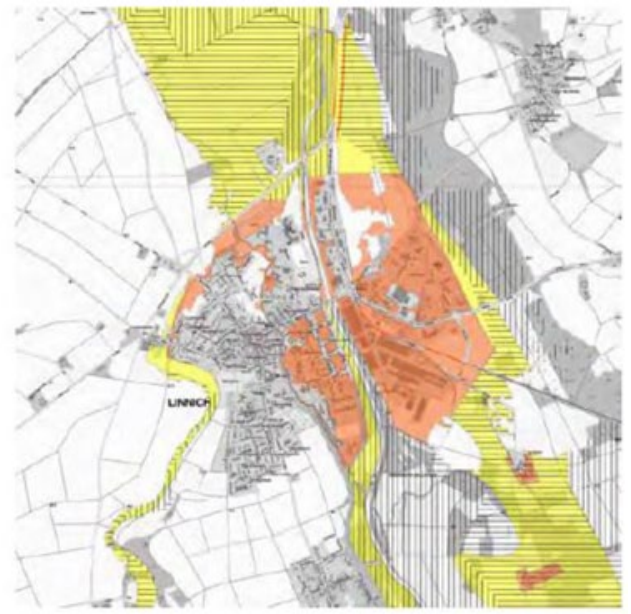

1 III FFH-Gebiet

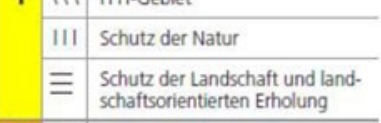

2 /I/ Grundwasserschutz-WSG und bei der Abwägungsentscheidung konkret zu verorten (Greiving 2002: 74; Faßbender 2012: 86). Deshalb kommt der Verwaltung bei der Ausfüllung von gesetzlich geregelten finalen Entscheidungsprogrammen ein Abwägungsspielraum nach dem Modell der planerischen Abwägung zu. Dies bedeutet, dass ein Spielraum für die jeweiligen Verfahren sowohl bei der Auswahl einer Analysemethode als auch bei der Bewertung der Ergebnisse und der Entscheidungsfindung besteht. Das Gewicht des Belanges ergibt sich bei einer Risikoanalyse aus der Kombination aus Eintrittswahrscheinlichkeit und der Konsequenz bestimmter Ereignisse.
Der Abwägungsspielraum besteht dann in der Entscheidung, ob ein bestimmbares Risiko in Kauf genommen werden soll, weil andere Belange vorgezogen werden, oder ob dies eben nicht akzeptiert wird. Dabei ist in der planerischen Begründung im Einzelnen transparent darzulegen, welche fachlichen Daten und Prognosen aus welchen Gründen herangezogen wurden. Bei diesem Schritt darf sich der Planungsträger nicht mit einer schematischen und abstrakten Beurteilung begnügen. Die Einschätzungen und Prognosen müssen vielmehr unter Heranziehen des jeweils gebotenen empirischen Materials plausibel sein. Dafür muss die 
Abb. 6 Risikokarte Hochwasser (eigene Darstellung; in BMVI/ BBSR 2015: 99). ASB Allgemeinen Siedlungsbereiche, GIB Bereiche für gewerbliche und industrielle Nutzungen
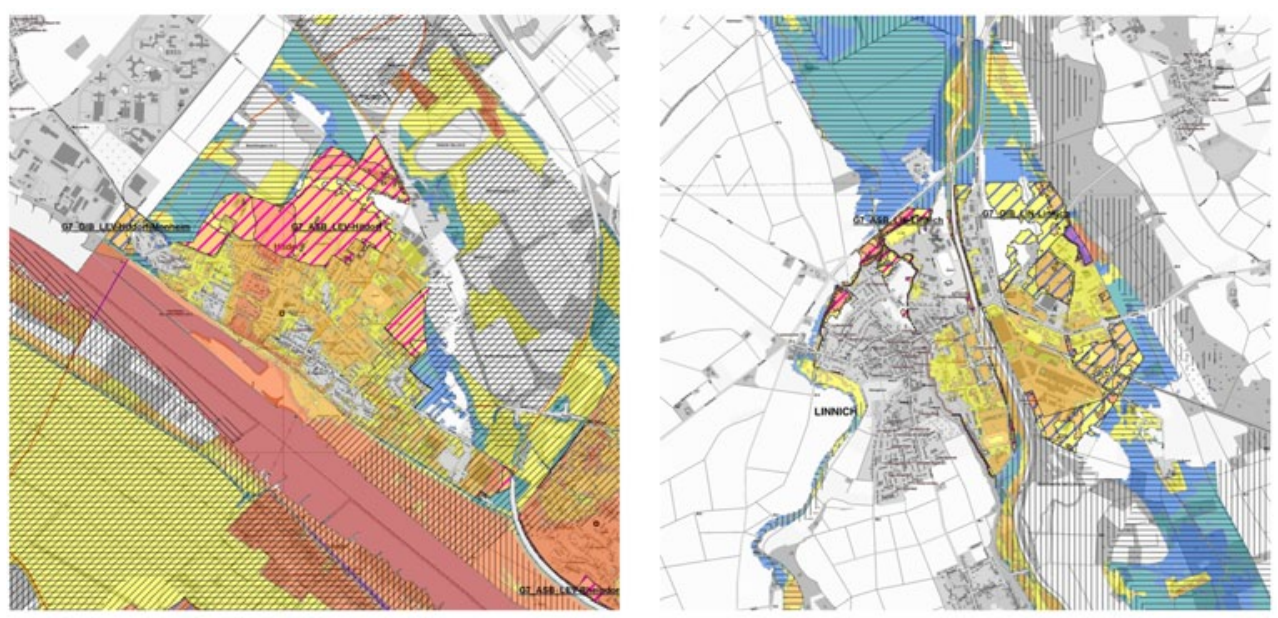

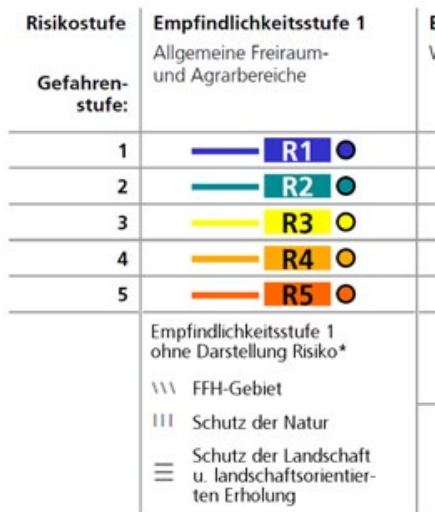

\begin{tabular}{l|l} 
Empfindlichkeitsstufe 2 & Empfindlichkeitsstufe 3
\end{tabular}

Soziale Infrastruktur von (aber)regionaler Bedeutung, Straßen und chienenwege (aberregionaler/großrăumiger Verkehr), Allgemeine Siedlungssplitter, Bereiche fur gewerbliche/industrielle Nutzungen

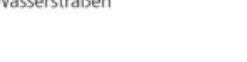

R3 O

$\begin{array}{r}\mathrm{R} 2 \mathrm{O} \\ \mathrm{R} 3 \mathrm{O} \\ \mathrm{R} 4 \mathrm{O} \\ \hline \mathrm{R} 5 \mathrm{O} \\ \hline \mathrm{R} 6 \\ \hline\end{array}$

R3 O

R4 O

R5 0

$\mathbf{R} 6 \mathbf{R}$

Empfindlichkeitsstufe 3

ohne Darstellung Risiko*

III Grundwasserschutz - WSG

IIII ASB, möglicherweise unbebaut IIII GIB, möglicherweise unbebaut

Far die Lesbarkeit der Karte wurde bei Raumfunktionen auf die differenzierte farbliche Darstellung des Risikos verzichtet methodische Herangehensweise der betreffenden Risikoanalyse erläutert und sich vom Planungsträger zu eigen gemacht werden (Faßbender 2012: 93). Der Ermittlung der Tatsachenbasis und der Konsistenz der methodischen Herangehensweise kommt dabei große Bedeutung für die Rechtssicherheit der planerischen Abwägung zu, die sich auf diese Methodik stützt.

Grundsätzlich bedeuten Unsicherheiten in der Analyse und Bewertung von Risiken aber nicht, dass kein Handlungserfordernis bzw. keine entsprechenden Handlungsmöglichkeiten bestehen würden. Auf diese Implikationen wird unter Abschnitt 5.6 näher eingegangen.

\section{Handlungsstrategien des planerischen Risikomanagements}

Grundsätzlich ist die Bewertung von Risiken Teil der planerischen Entscheidungsfindung bzw. der Abwägung der unterschiedlichen Belange. Gleichwohl ist es etablierter Bestandteil der Umweltprüfung gemäß $\S 9$ Abs. 1 ROG, Vermeidungs-, Minderungs- und Ausgleichsmaßnahmen zu ermitteln (vgl. Anhang I Buchstabe g der SUP-Richtlinie) (EU 2001). Der Planungsträger stellt die Ergebnisse des Umweltberichts in die Abwägung ein, innerhalb derer darüber zu befinden ist, ob die ermittelten Risiken akzeptiert werden, weil anderen Belangen, wie etwa der wirtschaftlichen Entwicklung, Vorrang eingeräumt wird, oder aber ob die im Umweltbericht dargestellten Vermeidungs-, Minderungs- und Ausgleichsmaßnahmen in entsprechende raumordnerische Festlegungen (z. B. Vorranggebiete) überführt werden.

\subsection{Risikovermeidungsstrategien}

Risikovermeidungsstrategien setzen im Wesentlichen auf den etablierten Trennungsgrundsatz: Gefährdete Bereiche sind von empfindlichen Raumnutzungen freizuhalten (vgl. Achtungsabstände nach Störfallverordnung, Vorranggebiet vorbeugender Hochwasserschutz etc.). Akteure dieser Strategien sind die Regionalplanung in Abstimmung mit den zuständigen Fachplanungen. Adressaten sind Kommunen und Anlagenbetreiber.

Diese Vorgehensweise ist beim Umgang mit dem Bestand an Siedlungsflächen aber bisher wenig etabliert. Bei Nutzungsänderungen bzw. bauleitplanerischer Aktivität gelten jedoch die Ziele der Raumordnung ebenso, sodass die Wiedernutzung einer Brache zu Siedlungszwecken im Überschwemmungsbereich unterbunden werden könnte. Der Flächennutzungsplan (FNP) muss gegebenenfalls an 
geänderte Ziele der Raumordnung nachträglich angepasst werden. Hier agiert die Regionalplanung im Rahmen einer Regionalplanfortschreibung im Gegenstromprinzip mit den Kommunen, die auch die Adressaten der geänderten raumordnerischen Festlegung sind.

Im Hinblick auf den Umgang mit dem baulichen Bestand ist das Planschadensrecht relevant, in dem der Bodenwertverlust geregelt wird. Erfolgt die Nutzungsänderung innerhalb der ersten sieben Jahre ab Nutzungszulässigkeit, bemisst sich demnach die Entschädigung nach der Differenz aus dem Bodenwert der früheren zur aktuellen Nutzungsmöglichkeit. Nach Ablauf der Siebenjahresfrist (§ 42 Abs. 2 BauGB) gilt nach $\S 42$ Abs. 3 BauGB, dass - von Ausnahmen abgesehen - eine seit mehr als sieben Jahren zulässige, aber nicht ausgeübte Nutzung nicht entschädigungspflichtig ist. Dennoch dürfte es Privaten wie Kommunen Probleme bereiten, wenn Flächen im Sinne einer Bevorratungspolitik erworben wurden, und es aufgrund der nachträglichen FNPAnpassungspflicht zu Nutzungsänderungen kommt, die die Buchwerte der Flächen, die als Bauerwartungsland im Vermögenshaushalt stehen, in Frage stellen würden. Reserven für Betriebserweiterungen besitzen meist schon ein entsprechendes Baurecht.

\subsection{Risikominderungsstrategien}

Risikominderungsstrategien können entweder an der Gefahrenquelle selbst, ihrer Ausbreitung im Raum oder an der Empfindlichkeit der Schutzgüter ansetzen:

- So kann Hochwasser über den Rückhalt von Wasser in der Fläche gemindert oder seine Ausbreitung im Raum über Retentionsflächen, Polder und Deiche beeinflusst werden. Hier ist eine Zusammenarbeit zwischen Regionalplanung, Wasserwirtschaft, Forstplanung, Landschaftsplanung und agrarstruktureller Fachplanung angezeigt.

- Störfälle können über Gefahrenpläne der Betreiber eingegrenzt bzw. in ihrer Ausbreitung im Raum beeinflusst werden. Zuständig sind hier die Anlagenbetreiber und die Gefahrenabwehrbehörden.

- Eine thermische Belastung von Stadtregionen kann über die Verbesserung von Luftaustauschbeziehungen (etwa die Festlegung zusätzlicher regionaler Grünzüge) eine Minderung erfahren. Auf der Ebene der Bauleitplanung ist dies dann über die Freihaltung innerstädtischer Grünflächen möglich.

Besonders empfindliche bzw. schutzwürdige Raumnutzungen wie kritische Infrastrukturen könnten aus gefährdeten Bereichen ausgeschlossen werden: Ein Vorbehaltsgebiet könnte mit einem Vorrang (bzw. Ausschluss) für diese Nutzung verbunden werden. So wäre eine Errichtung in Gefährdungsräumen nur bei Nachweis eines Gebiets- und Objektschutzes auch bei Extremereignissen wie beispielsweise dem 500-jährlichen Hochwasser zulässig. Damit wird ein höheres Schutzziel als für andere Raumfunktionen/-nutzungen erreicht. Akteur wäre hier wiederum die Regionalplanung, Adressaten die Kommunen, aber auch Infrastrukturbetreiber.

\subsection{Risikoausgleichsstrategien}

In der planerischen Gesamtabwägung kann es legitim sein, ein Risiko um eines Vorteils willen in Kauf zu nehmen: Eine Siedlungsentwicklung in deichgeschützten Gebieten mag angesichts fehlender Alternativstandorte erforderlich sein, um kommunale Flächenbedarfe zu realisieren. Denkbar wäre jedoch eine Kompensationspflicht, etwa in Form der Renaturierung von brach gefallenen Nutzungen hinter den Deichen (als Ziel anstelle eines Grundsatzes).

Die Innenverdichtung dient dem Schutz des Freiraums und der Verkehrsvermeidung, kann aber das Stadtklima negativ beeinflussen, da bebaute und versiegelte Flächen zum Wärmeinseleffekt beitragen. Die Festlegung zusätzlicher innerstädtischer Siedlungsflächen in klimarelevanten Gebieten könnte mit einer Kompensationspflicht versehen werden, die etwa als Ausgleich auf die Schaffung neuer, Überwärmungstendenzen kompensierender Grün- oder Wasserflächen abzielt.

Bei Planungen, die die Risiken für andere Kommunen erhöhen, sollte eine Ausgleichspflicht über eine textliche Festlegung konstituiert werden. Bei vorhabenbezogenen Planungen (nur hier ist das konkrete Projekt bekannt) müssen die Interessen der Nachbarkommunen stärker Berücksichtigung finden. Die Umsetzung kann über einen landesplanerischen Vertrag erfolgen. Die Ausgleichspflicht soll jedoch nicht zu einer „Doppelbestrafung“ führen, indem die negativ betroffene Kommune z. B. zusätzlich naturschutzgesetzliche Kompensationsflächen für das Kraftwerk bereitstellen muss; es sei denn, diese sieht Vorteile in der Flächenbereitstellung. Im Hinblick auf vertragliche Regelungen der Ausgleichspflicht sind zudem die Beziehungen zwischen Vorhabenträger und bevorteilten bzw. benachteiligten Kommunen hinsichtlich des Risikoausgleichs zu klären, aber auch bezüglich anderer Tauschgüter und -ressourcen, die für einen realistischen Ausgleichsmechanismus notwendig erscheinen.

Hinzu kommt die allen vorgenannten Strategien innewohnende Risikoübertragungsstrategie, bei der Risiken im eigenen Plan- oder Kompetenzbereich zu Lasten anderer Plan- oder Kompetenzbereiche vermieden oder minimiert werden. Klassisches Beispiel ist hier die Ober-Unterlieger-Problematik. Diese ruft nach einer überörtlichen Bewältigung im Rahmen der Raumordnung.

Risikoausgleichsstrategien eignen sich vor allem für diejenigen Bundesländer, in denen keine positiv-allokative 
Standortsteuerung im Bereich der Siedlungsflächenentwicklung stattfindet. Im Falle einer Standortsteuerung (Nordrhein-Westfalen, Hessen, Region Hannover, Verband Region Stuttgart, Großraum Berlin) kann die Regionalplanung die kumulative Risikobelastung eines Raums in die Allokation von Siedlungsflächen einbeziehen. Ohne diese Steuerungsoption verbleiben den Planungsträgern nur Mengengerüste oder negativ-restriktive Steuerungsansätze. Diese beziehen sich bisher aber immer auf einzelne Gefahren (z. B. Hochwasser oder gravitative Massenbewegungen).

\subsection{Umgang mit Bestand und Planung}

Aus der Perspektive der Raumordnung zielen mögliche Handlungsoptionen im Risikomanagement sowohl auf den Bestand als auch die Planung von künftigen Raumnutzungen und kritischen Infrastrukturen. Die Gebietskategorien sind daher grundsätzlich an der Steuerungsfähigkeit des Regionalplans orientiert.

Hieraus ergeben sich unterschiedliche Anwendungsfälle für die Regionalplanung und die Frage nach dem Umgang mit möglichen planerischen Handlungsansätzen. Im Bestand ist Folgendes zu hinterfragen:

- Sind empfindliche Raumnutzungen und Funktionen mit Schutzzielen hinsichtlich der unterschiedlichen Gefahren ausgestattet? Gibt es differenzierte Festlegungen für unterschiedliche Anforderungen (kritische Infrastrukturen)?

- Wie können die Schutzzielanforderungen mit regionalplanerischen Festlegungen verbunden werden?

- Welche Folgenutzungen sind bei Nutzungsänderung (Brachfallen) unter Berücksichtigung des gegebenen Risikoprofils sinnvoll?

Bei Neuplanungen sollte die Nutzungssteuerung nach dem Trennungsgrundsatz erfolgen. Die planerische Frage ist hierbei, ob eine Planungsabsicht zu einem höheren Risiko führen würde und daher gegebenenfalls zu unterbinden oder in ihren Folgen zu mindern sei. Hierzu ist im Vorfeld eine Analyse der Gefahrensituation notwendig. Dabei ist abzuwägen, ob angesichts der spezifischen Empfindlichkeit und Schutzwürdigkeit in Betracht kommender Raumnutzungen (wie z. B. allgemeine Siedlungsfläche) eine entsprechende planerische Inanspruchnahme, mit der eine Erhöhung des bestehenden Risikos verbunden wäre, in der Abwägung gerechtfertigt werden kann. Insofern ist für Neuplanungen nicht das Risiko, sondern die Gefährdung maßgeblich für die Strategieentwicklung, da eine Risikokarte - auf Realnutzung fußend - gegebenenfalls ein geringes Risiko für ein Plangebiet aufzeigen kann. Hier ist abzuschätzen, inwieweit eine Neuplanung zu einer Risikoerhöhung führen würde.

\subsection{Umsetzung und Wirksamkeit}

In Bezug auf die Wirksamkeit ist zu fragen, wie die Ziele und Grundsätze von den Planadressaten (d. h. Kommunen und anderen öffentlichen Planungsträgern) umgesetzt werden sollten und wie diese Umsetzung nachprüfbar ausgestaltet werden kann. Hierbei bieten sich Monitoringsysteme in der Raumordnung an, die ohnehin für die Überwachung unvorhersehbarer negativer Umweltauswirkungen in der Umweltprüfung benötigt werden. In diesem Kontext ist auch das Entstehen neuer Risiken prinzipiell als eine solche unvorhergesehene Umweltauswirkung zu verstehen, für die Abhilfemaßnahmen getroffen werden müssten.

\subsection{Alternative Strategien in raumplanerisches Handeln integrieren}

Für die mit Risiken und deren Komponenten - Eintrittswahrscheinlichkeit und Ausmaß von Schäden - verbundene Unsicherheit (vergleiche dazu Kapitel 4.3) stehen konzeptionell alternative Strategien zur Verfügung, die stärker in das Instrumentarium der Raumplanung einbezogen werden sollten und über das traditionelle Vorsorgeprinzip hinausgehen. Hallegatte (2008: $244 \mathrm{f}$.) fasst neuere Ansätze in fünf Strategien zusammen (vgl. auch BMVBS/ BBSR 2013b: 34 f.):

- „No-Regret-Strategien“, die einen Mehrwert erbringen unabhängig vom Eintreffen projizierter Klimawandelfolgen (wie beispielsweise einer Zunahme von Starkregenereignissen), mit denen auch eine hohe Unsicherheit verbunden ist. Dieser Strategietyp eignet sich auch für andere Risiken, die etwa mit technischen Störfällen verbunden sind, deren Eintrittswahrscheinlichkeit nicht bestimmbar ist.

- Reversible Strategien, die einen flexibleren Umgang mit Unsicherheit erlauben. Übersetzt in die Logik der Raumordnung bedeutet dies (so auch BMVBS 2011: 5), Festlegungen primär in Form von Grundsätzen der Raumordnung oder als Planungshinweise für Kommunen und Fachplanungen zu treffen, die noch nicht letztabgewogen sind und damit reversibel bleiben.

- Strategien, die Entscheidungshorizonte verringern und mittelfristige Lösungen anstreben. Diese setzen auf planerisches Handeln, das sich am Vorsorgeprinzip ausrichtet ( 1 Abs. 1 Nr. 2 ROG). Deshalb kann es sinnvoll sein, künftig nicht den gesamten nachgewiesenen Bedarf von Siedlungsflächen als Kontingent zur Verfügung zu stellen oder allokativ im Regionalplan darzustellen. Dies entspricht dem Konzept der sequenziellen Realisierung von Planinhalten. Die weiteren Entscheidungsstufen werden offen gehalten und dann ausgeführt, wenn Dringlichkeit besteht bzw. die Unsicherheit der vorliegenden 
Informationen überwunden ist. Ähnlich argumentiert Janssen (2012: 110), wenn er das durch Monitoring, Bewertung und Nachbesserung erlangte Wissen als Chance ansieht, immer neue Informationen in das Verwaltungsverfahren einzubringen und Verwaltungsakte eingeschränkt dynamischer zu gestalten.

- „Sanfte“ Strategien, um auf (meist aufwendige und teure) technische Lösungen verzichten zu können und stattdessen auf kooperativen Ansätzen aufzubauen. Innerhalb von Prozessen kooperativer Regionalentwicklung kann auch bei Entscheidungen unter Ungewissheit ein Konsens als funktionales Äquivalent rechtlicher Normierung dienen.

- „Safety margin strategies“, die sich auf Maßnahmen mit „Sicherheitszuschlägen“ beziehen. Hier besteht ein enger Zusammenhang zum Vorsorgeprinzip. „Safety margin“Strategien werden etwa in Bayern bei der Planung wasserbaulicher Anlagen wie Hochwasserdämmen vorgesehen: Es wird ein „Klimazuschlag“ von $15 \%$ auf das Bemessungsereignis (HQ100) aufgesetzt (LfU 2005: 1). Diese Zuschläge sind nur dann begründbar, wenn sie sich aus einer Evidenzbasis ableiten lassen und differenziert für die einzelnen Gewässerabschnitte erfolgen, weil die Auswirkungen des Klimawandels räumlich differenziert auftreten.

\section{Fazit}

\subsection{Diskussion}

Aus dem Spektrum raumrelevanter Risiken konnten nicht alle Gefahrenkomplexe beleuchtet werden. Gründe hierfür lagen z. B. in der mangelnden Datenverfügbarkeit (Beispiel Hitze) oder auch im Ressourcenansatz für das MORO selbst. Für die Gefahrenkomplexe Erdbeben, Flusshochwasser und technische Störfälle wurden die schutzgutbezogenen Risiken systematisch bearbeitet. Hier brachte das MORO „Vorsorgendes Risikomanagement in der Regionalplanung“ neue Ansätze, die bislang in dieser Form in der Regionalplanung keine Berücksichtigung fanden.

Generell stellt sich die Frage, ob angesichts der relativ geringen Eintrittswahrscheinlichkeit für schadensträchtige Erdbeben in Deutschland das Risiko hoch genug ist, um überhaupt regionalplanerische Regelungen treffen $\mathrm{zu}$ müssen. Primär gilt es, Baunormen so zu gestalten, dass Gebäude und Infrastrukturen einem Erdbeben standhalten. Baurechtliche Genehmigungen sind daher ein Ansatzpunkt, um wirksam Risikovorsorge zu betreiben. Im Regionalplan könnte ein Grundsatz zum erdbebenangepassten Bauen (analog zum Hochwasser) die Kommunen dazu anhalten, sich mit dem Thema Erdbeben auseinanderzusetzen. Weitergehende Einschränkungen sind im Bereich besonders schutzbedürftiger (z. B. kritischer) Infrastrukturen bzw. bei aus sich selbst heraus besonders gefährlichen Nutzungen (z. B. Störfallbetriebe) denkbar.

Bei Flusshochwasser wurden nicht nur die Wiederkehrintervalle, sondern auch die Gefahrenintensität in die Risikobetrachtung einbezogen. Zudem wurden auch risikobehaftete, deichgeschützte Bereiche integriert. In diesem Themenfeld konnte der Bedarf für eine differenzierte Betrachtung empfindlicher Strukturen am deutlichsten herausgearbeitet werden. Neu ist dabei der Vorschlag, besonders kritische Infrastrukturen im Rahmen der regionalplanerischen Festsetzungen in deichgeschützten Bereichen mit Hochwasserrisiken auszuschließen. Ein Defizit der Risikobetrachtung liegt darin, dass die Gefahr des Deichbruchs oftmals nicht betrachtet wird. Seitens der Wasserwirtschaft besteht die Möglichkeit der Integration von Deichbruchszenarien. Allerdings lassen sich dennoch keine Eintrittswahrscheinlichkeiten bestimmen, was eine Einordnung in eine Gefahrenstufe schwierig macht. Im Hinblick auf Schadensausmaß und Vulnerabilität sollten auch Funktionsbeeinträchtigungen von Raumstrukturen im Schadensfall ermittelt werden. Dies sind beispielsweise bei Hochwasser nicht überschwemmte Bereiche, die aber nicht mehr erreichbar sind. Insofern sind die Auswirkungen von Überflutung auch im Verkehrsnetz relevant. Das Thema Siedlungsrückzug wurde in der Modellregion nicht vertiefend betrachtet, würde sich aber gerade in schrumpfenden Regionen anbieten, um Synergien im Kontext einer Bewältigung des demographischen Wandels zu nutzen, da der Rückbau über die Stadtumbauprogramme förderfähig wäre.

Auch bei der Betrachtung technischer Störfälle konnte das Modellvorhaben die Bedeutung dieses für die Regionalplanung neuen Themenfelds in der Risikovorsorge verdeutlichen. Es zeigte sich, dass Fachnormen wie die Seveso-III-Richtlinie alleine nicht ausreichen, um die regionalplanerischen Belange der Risikobewertung zu bedienen. So ist die Störfallbewertung nicht mit der Risikologik vergleichbar. Ein Störfall ist ein zeitlich begrenztes Ereignis, das für den Menschen gesundheitsschädlich sein kann. Dabei wird keine Differenzierung vorgenommen, ob es sich bei der betroffenen Nutzung um einen Kindergarten oder um ein Hotel handelt, und es wird vor allem keine Wahrscheinlichkeit, sondern lediglich im Sinne eines Szenarios ein sogenannter „DennochStörfall“ bestimmt. Aus der Sicht der Risikoforschung ist daher der in Deutschland vorherrschende deterministische Ansatz zur Ermittlung angemessener Abstände nicht hinreichend, da letztendlich weder eine Gefährdung im Sinne einer Frequenz-Magnitude-Beziehung bestimmt, noch die Empfindlichkeit verschiedener Schutzgüter betrachtet und damit am Ende auch kein Risiko bestimmt wird. Damit fehlt die Grundlage für eine gesellschaftliche Debatte im 
Sinne des Risiko-Governance-Ansatzes über akzeptable Risiken für unterschiedliche Schutzgüter, die gegebenenfalls unterschiedlich schutzbedürftig sind (Glade/Greiving 2011). Dass eben diese Debatte Not tut, zeigt die anhaltende Kontroverse um die CO-Pipeline in der Region Düsseldorf (Bezirksregierung Düsseldorf 2015). Letztendlich fordern die Gegner eine Debatte über Risiken und deren Akzeptabilität, die innerhalb der vorgesehenen Verfahrensschritte der Planfeststellung nicht geführt werden kann. Ein weiteres Problem besteht darin, dass der Leitfaden der Kommission für Anlagensicherheit (KAS 2010) und die Seveso-IIIRichtlinie nicht nur vom Menschen, sondern auch von der Umwelt als Schutzgüter sprechen. Allerdings gibt es europaweit nur für den Menschen begründete Faktoren, um Gesundheitsgefahren zu bestimmen und auf deren Grundlage „Achtungsabstände“ bzw. „angemessene Abstände“ und „Maßnahmen“ festzulegen. Für andere Schutzgüter wie Flora, Fauna oder Gewässer gibt es derzeit keine Kennwerte, mit denen man relevante Abstände bestimmen könnte. Über die so genannten „Seveso-Betriebe“ (aus sich selbst heraus gefährliche Einrichtungen oder „Störfallbetriebe") hinaus sollten zukünftig auch Atomanlagen oder Rohrfernleitungen in die Risikobetrachtung einbezogen werden. Linienhafte Strukturen wie Pipelines konnten im Rahmen des MORO aufgrund der mangelnden Datenverfügbarkeit nicht bearbeitet werden, wären aber grundsätzlich raumordnerisch relevant.

Sowohl für die Identifikation thermischer Belastungen in Stadtregionen als auch für eine Definition von Schwellenwerten oder die Ermittlung des Einflusses von Luftaustauschprozessen sind Grundlageninformationen unerlässlich. Da es keine zuständige Fachplanung gibt, bestehen hier in der Regel Defizite. Regionalplanerisch ist das Thema von Bedeutung, da das Paradigma der Innenentwicklung und die damit verbundene Zunahme der Baumassen zu einer Erhöhung der Hitzebelastung (auch während Hitzewellen) beitragen können. Zudem kann nur die überörtliche Regionalplanung Luftleitbahnen planerisch angemessen sichern, da sich diese in Agglomerationsräumen häufig auf dem Gebiet der Umlandgemeinden befinden. Vor Ort mögen diese Freiräume lokal-klimatisch keine entsprechende Bedeutung besitzen, sodass eine mögliche Inanspruchnahme für Siedlungszwecke aus regionalplanerischer Perspektive verhindert werden muss. Hier ist also eine regionale Risikoausgleichsstrategie zwingend erforderlich, die in Analogie zum Oberlieger-Unterlieger-Problem im Hochwasserschutz zu sehen ist. Eine solche Risikoausgleichsstrategie wäre insbesondere in Ländern ohne positiv-allokative Standortsteuerung wichtig, um die Inanspruchnahme dieser Flächen als Siedlungsfläche zu verhindern. Grundsätzlich besteht bei diesem Gefahrenkomplex auch ein Zielkonflikt mit der gängigen Leitvorstellung kompakter Siedlungsentwicklung. Hier müsste im Sinne eines Regel-Ausnahme-Verhält- nisses gegebenenfalls eine weitere Verdichtung nachweislich thermisch beeinträchtigter Siedlungsflächen zugunsten einer Außenentwicklung abgewendet werden. Auch wenn Innenentwicklungspotenziale vorhanden sind, können diese in Abwägung mit dem lokalen Klimakomfort aufgegeben werden.

\subsection{Schlussfolgerungen}

Die Ergebnisse des Modellvorhabens verdeutlichen die Möglichkeiten, aber auch die Herausforderungen und Fallstricke der Risikovorsorge in der räumlichen Planung. Vieles hängt von der Datenverfügbarkeit, aber auch den personellen Ressourcen eines Regionalplanungsträgers ab. Gleichwohl muss mit Verweis auf die erweiterte Sichtweise der Umweltprüfung betont werden, dass die Behandlung von Katastrophenrisiken zukünftig eine Pflichtaufgabe darstellen wird.

An Grenzen stößt grundsätzlich die Berücksichtigung kritischer Infrastrukturen: Während deren baulich-räumliche Komponente in einer Risikoanalyse erfasst werden kann, ist eine Behandlung der systemischen Bedeutung einzelner Infrastrukturen kaum möglich, da Regionalplanung einen räumlichen Zuständigkeitsbereich hat, während die kaskadenartigen Folgen im Falle des Ausfalls von Infrastrukturen nicht an administrativen Grenzen Halt machen. Dies unterstreicht zwar ihre besondere Schutzwürdigkeit, lässt aber eine regionalplanerische Risikoanalyse kaum zu, da ein Planungsträger unmöglich alle denkbaren Ausfallszenarien mit unter Umständen globalen Auswirkungen auf Lieferketten oder Transportwege im Blick haben kann.

Die methodischen Ansätze der Risikoanalyse wurden erprobt und regionsspezifisch angepasst. Insgesamt lässt sich das Vorgehen der Risikoanalyse gut in die Prüfschritte einer Umweltprüfung wie auch in die vorbereitenden Arbeiten zum Regionalplan integrieren. Dabei zeigte sich die Bedeutung dialogischer Verfahren und mithin des Risiko-Governance-Ansatzes im Rahmen des MORO. So wurden in der Modellregion die Bewertungen zu Gefahrenund Empfindlichkeitsstufen zwischen der Regionalplanung und den Fachressorts sowie der Forschungsassistenz intensiv diskutiert. Diese Vorgehensweise hat die Zusammenarbeit und den Austausch zwischen den Dezernaten stark befördert. Die transparente Darstellung und Kommentierung der Genese der einzelnen Bearbeitungsschritte war eines der zentralen Anliegen der Bezirksregierung, um für alle Seiten einen größtmöglichen Nutzen und Lerneffekt erzielen zu können. Ein vergleichbares Vorgehen wird daher auch für andere Planungsregionen empfohlen. 


\section{Literatur}

Battis, U.; Moench, C.; Uechtritz, M.; Mattes, C.; von der Groeben, C. (2015): Gutachterliche Stellungnahme zur Umsetzung der UVP-Änderungsrichtlinie im Baugesetzbuch im Auftrag des BBSR. http://www.bmub.bund.de/fileadmin/Daten_BMU/Download_PDF/Staedtebaurecht/baugb_gutachten_uvp_aendrl_bf.pdf (29.2.2016).

BBK - Bundesamt für Bevölkerungsschutz und Katastrophenhilfe (Hrsg.) (2009): Vulnerabilität Kritischer Infrastrukturen. www. bbk.bund.de/SharedDocs/Downloads/BBK/DE/Publikationen/ PublikationenForschung/FiB_Band4.pdf? blob = publicationFile (08.06.2015). Bonn. $=$ Forschung im Bevölkerungsschutz, 4.

BBK - Bundesamt für Bevölkerungsschutz und Katastrophenhilfe (Hrsg.) (2010): Methode für die Risikoanalyse im Bevölkerungsschutz. Bonn.

Bezirksregierung Düsseldorf (Hrsg.) (2015): CO-Pipeline. www. bezreg-duesseldorf.nrw.de/umweltschutz/immissionsschutz/COPipeline/index.jsp (21.08.2015).

Birkmann, J.; Greiving, S.; Overbeck, G.; Pohl, J.; Rother, K.-H.; Rumberg, M.; Spangenberg, M.; Wernig, R.; Zehetmair, S. (2011): Zukünftige Ausgestaltung des Risikomanagements in der Raumplanung. Hannover. $=$ Positionspapier der ARL, 86 .

BMI - Bundesministerium des Inneren (Hrsg.) (2009): Nationale Strategie zum Schutz Kritischer Infrastrukturen (KRITISStrategie). Berlin.

BMVBS - Bundesministerium für Verkehr, Bau und Stadtentwicklung (Hrsg.) (2011): Vulnerabilitätsanalyse in der Praxis. Inhaltliche und methodische Ansatzpunkte für die Ermittlung regionaler Betroffenheiten. BMVBS-Online-Publikation 21/2011. www.bbsr. bund.de/BBSR/DE/Veroeffentlichungen/BMVBS/Online/2011/ ON212011.html (20.08.2012).

BMVBS - Bundesministerium für Verkehr, Bau und Stadtentwicklung; BBSR - Bundesinstitut für Bau-, Stadt- und Raumforschung (Hrsg.) (2013a): Heute Zukunft gestalten. Raumentwicklungsstrategien zum Klimawandel. Berlin. www.klimamoro.de/ fileadmin/Dateien/Ver\%C3\%B6ffentlichungen/Publikatione_ aus dem Modellvorhaben/Barrierefreie Version KlimaMORO Broschuere_web.pdf (12.01.2016).

BMVBS - Bundesministerium für Verkehr, Bau und Stadtentwicklung; BBSR - Bundesinstitut für Bau-, Stadt- und Raumforschung (Hrsg.) (2013b): Methodenhandbuch zur regionalen Klimafolgenbewertung in der räumlichen Planung. Berlin/Bonn. www.bbsr.bund.de/BBSR/DE/FP/MORO/Studien/2011/Leitfaden RegionaleKlimafolgenbewertung/Downloads/DL_Handbuch.pdf (11.12.2013).

BMVBS - Bundesministerium für Verkehr, Bau und Stadtentwicklung; BBSR - Bundesinstitut für Bau-, Stadt- und Raumforschung (Hrsg.) (2014): Regionale Fragestellungen - regionale Lösungsansätze. Ergebnisbericht der Vertiefungsphase des Modellvorhabens der Raumordnung „Raumentwicklungsstrategien zum Klimawandel" (KlimaMORO). BMVBS-Online-Publikation 01/2014. www.bbsr.bund.de/BBSR/DE/Veroeffentlichungen/BMVBS/ Online/2014/DL_ON012014.pdf;jsessionid $=$ B7984DD542ED4 3103007A8EACCB4FD72.live2053? _blob = publicationFile\&v $=2(12.01 .2016)$.

BMVI - Bundesministerium für Verkehr und digitale Infrastruktur; BBSR - Bundesinstitut für Bau-, Stadt- und Raumforschung (Hrsg.) (2015): Vorsorgendes Risikomanagement in der Regionalplanung. Berlin/Bonn. Im Druck.

Davy, B. (1990): Gefahrenabwehr im Anlagenrecht. Wien, New York. $=$ Forschungen aus Staat und Recht, 91 .

EEA - European Environment Agency (Hrsg.) (2012): Climate change, impacts and vulnerability in Europe 2012. An indicatorbased report. Kopenhagen.
Einig, K. (2011): Funktion und Folgen von Modellvorhaben für die Politikberatung. In: Informationen zur Raumentwicklung 7-8/2011, 435-451.

EU - Europäische Union (2001): Richtlinie 2001/42/EG des Europäischen Parlaments und des Rates vom 27. Juni 2001 über die Prüfung der Umweltauswirkungen bestimmter Pläne und Programme. http://eur-lex.europa.eu/LexUriServ/LexUriServ.do? uri=OJ:L:2001:197:0030:0037:DE:PDF (01.01.2016).

EU - Europäische Union (2007): Richtlinie 2007/60/EG des Europäischen Parlaments und des Rates vom 23. Oktober 2007 über die Bewertung und das Management von Hochwasserrisiken. http://eur-lex.europa.eu/legal-content/DE/TXT/PDF/?uri=CELE $\mathrm{X}: 32007 \mathrm{~L} 0060 \&$ from=DE (23.06.2013).

EU - Europäische Union (2012): Richtlinie 2012/18/EU des Europäischen Parlaments und des Rates vom 4. Juli 2012 zur Beherrschung der Gefahren schwerer Unfälle mit gefährlichen Stoffen, zur Änderung und anschließenden Aufhebung der Richtlinie 96/82/EG des Rates. http://eur-lex.europa.eu/LexUriServ/ LexUriServ.do?uri=OJ:L:2012:197:0001:0037:DE:PDF (05.06.2014)

EU - Europäische Union (2014): Richtlinie 2014/52/EU des Europäischen Parlaments und des Rates vom 16. April 2014 zur Änderung der Richtlinie 2011/92/EU über die Umweltverträglichkeitsprüfung bei bestimmten öffentlichen und privaten Projekten. http://eur-lex.europa.eu/legal-content/DE/TXT/PDF/?uri=CELE $\mathrm{X}: 32014 \mathrm{~L} 0052 \&$ from $=\mathrm{EN}(04.11 .2014)$.

Faßbender, K. (2012): Rechtsgutachten zu den Anforderungen an regionalplanerische Festlegungen zur Hochwasservorsorge erstattet im Auftrag des Regionalen Planungsverbands Oberes Elbtal/Osterzgebirge. Leipzig.

Field, C. B.; Barros, V.; Stocker, T. F.; Qin, D.; Dokken, D. J.; Ebi, K. L.; Mastrandrea, M. D.; Mach, K. J.; Plattner, G.-K.; Allen, S. K.; Tignor, M.; Midgley, P. M. (2012): Managing the Risks of Extreme Events and Disasters to Advance Climate Change Adaptation. A Special Report of Working Groups I and II of the Intergovernmental Panel on Climate Change. Cambridge, New York.

Glade, T.; Greiving, S. (2011): Naturgefahren und -risiken. Risikomanagement und Governance. In: Bevölkerungsschutz 2/2011, $13-19$.

Greiving, S. (2002): Räumliche Planung und Risiko. München.

Greiving, S. (2004): Risk Assessment and Management as an Important Tool for the EU Strategic Environmental Assessment. In: DISP 40, $157,11-17$.

Greiving, S. (2005): Der rechtliche Umgang mit Risiken aus Naturund Technikgefahren - von der klassischen Gefahrenabwehr zum Risk Governance? In: Zeitschrift für Rechtsphilosophie 02/2005, 53-61.

Greiving, S. (2011): Methodik zur Festlegung raum- und raumplanungsrelevanter Risiken. In: Pohl, J.; Zehetmair, S. (Hrsg.) (2011): Risikomanagement als Handlungsfeld in der Raumplanung. Hannover, 22-30. = ARL Arbeitsmaterial, 357.

Greiving, S.; Mayer, J.; Pohl, J.; Seiffert, J.; Bell, R. (2012): Kooperation zwischen Raumforschung und Raumplanungspraxis diskutiert am Beispiel der Berücksichtigung von Hangrutschungsgefährdungen im Regionalplan Neckar-Alb. In: RaumPlanung 158/159, 274-281.

Greiving, S.; Spangenberg, M.; Zehetmair, S. (2011): Raumstrukturkonzepte und ihr Verhältnis zur Risikoanfälligkeit. In: Pohl, J.; Zehetmair, S. (Hrsg.) (2011): Risikomanagement als Handlungsfeld in der Raumplanung. Hannover, 31-44. = ARL Arbeitsmaterial, 357.

Greiving, S.; Zebisch, M.; Schneiderbauer, S.; Lindner, C.; Lückenkötter, J.; Fleischhauer, M.; Buth, M.; Kahlenborn, W.; Schauser, I. (2015): A consensus based vulnerability assessment to climate change in Germany. In: International Journal of Climate Change Strategies and Management 7, 3, 306-326. 
Hallegatte, S. (2008): Strategies to adapt to an uncertain climate change. In: Global Environmental Change 19, 2, 240-247.

Janssen, G. (2012): Rechtsinstrumente der Klimaanpassung. In: Birkmann, J.; Schanze, J.; Müller, P.; Stock, M. (Hrsg.) (2012): Anpassung an den Klimawandel durch räumliche Planung Grundlagen, Strategien, Instrumente. Hannover, 106-120. = E-Paper der ARL, 13.

KAS - Kommission für Anlagensicherheit (Hrsg.) (1999): Schadensbegrenzung bei Dennoch-Störfällen. Empfehlungen für Kriterien zur Abgrenzung von Dennoch-Störfällen und für Vorkehrungen zur Begrenzung ihrer Auswirkungen. http://www.kas-bmu.de/ publikationen/sfk/sfk_gs_26.pdf (02.02.2016).

KAS - Kommission für Anlagensicherheit (Hrsg.) (2010): Leitfaden Empfehlungen für Abstände zwischen Betriebsbereichen nach der Störfall-Verordnung und schutzbedürftigen Gebieten im Rahmen der Bauleitplanung - Umsetzung § 50 BImSchG. www.kas-bmu. de/publikationen/kas/KAS_18.pdf (30.08.2014).

Klinke, A.; Renn, O. (2002): A New Approach to Risk Evaluation and Management: Risk-Based, Precaution-Based and DiscourseBased Strategies. In: Risk Analysis 22, 6, 1071-1094.

Krings, S. (2010): Verwundbarkeitsassessment der Strom- und Trinkwasserversorgung gegenüber Hochwasserereignissen. In: BBK - Bundesamt für Bevölkerungsschutz und Katastrophenhilfe (Hrsg.): Abschätzung der Verwundbarkeit gegenüber Hochwasserereignissen auf kommunaler Ebene. Bonn, 24-50.

LfU - Landesamt für Umwelt Bayern (Hrsg.) (2005): Gewässerkundlicher Jahresbericht für Bayern. Sonderthema Klimaänderungsfaktoren bei Planungen für den Hochwasserschutz. www.lfu. bayern.de/wasser/gewaesserkundliche berichte/sonderberichte/ doc/sb_klimaaenderungsfaktoren_bei_planung_hwschutz.pdf (13.01.2016).

Löfstedt, R. E. (2005): Risk Management in Post-Trust Society. London, Basingstoke $\mathrm{u}$. a.

Mickwitz, P.; Aix, F.; Beck, S.; Carss, D.; Ferrand, N.; Görg, C.; Jensen, A.; Kivimaa, P.; Kuhlicke, C.; Kuindersma, W.; Máñez, M.; Melanen, M.; Monni, S.; Branth Pedersen, A.; Reinert, H.; van Bommel, S. (2009): Climate Policy Integration, Coherence and Governance. Helsinki. = PEER Report, 2.

MKRO - Ministerkonferenz für Raumordnung (Hrsg.) (2013): Umlaufbeschluss „Raumordnung und Klimawandel“vom 06.02.2013. www. bmvi.de/SharedDocs/DE/Anlage/StadtUndLand/LaendlicherRaum/ mkro-handlungskonzept-klima.pdf? blob=publicationFile (13.03.2014).

Pohl, J. (2011): Risikovorsorge, Risikonachsorge und Raumplanung. In: Pohl, J.; Zehetmair, S. (Hrsg.) (2011): Risikomanagement als Handlungsfeld in der Raumplanung. Hannover, 11-20. = ARL Arbeitsmaterial, 357.

Reckien, D.; Flacke, J.; Dawson, R. J.; Heidrich, O.; Olazabal, M.; Foley, A.; Hamann, J. J.-P.; Orru, H.; Salvia, M.; de Gregorio Hurtado, S.; Geneletti, D.; Pietrapertosa, F. (2014): Climate change response in Europe: What's the reality? Analysis of adaptation and mitigation plans from 200 urban areas in 11 countries. In: Climatic Change 122, 1-2, 331-340.
Renn, O. (2008): Risk Governance - coping with uncertainty in a complex world. London.

Regionaler Planungsverband Oberes Elbtal/Osterzgebirge (Hrsg.) (2015): Vorentwurf 2015 zum Regionalplan 2016. Radebeul. www.rpv-elbtalosterz.de/index.php?id=fortschreibung_rpl (11.12.2015).

Riegel, C. (2015): Die Berücksichtigung des Schutzes Kritischer Infrastrukturen in der Raumplanung. Zum Stellenwert des KRITIS-Grundsatzes im Raumordnungsgesetz. Dissertation. https://publications.rwth-aachen.de/record/479433/files/479433. pdf (13.01.2016). = Berichte des Instituts für Stadtbauwesen und Stadtverkehr der RWTH Aachen, 59.

Sapountzaki, K.; Wanczura, S.; Casertano, G.; Greiving, S.; Xanthopoulos, G.; Ferrara, F. (2011): Disconnected policies and actors and the missing role of spatial planning throughout the risk management cycle. In: Natural Hazards 59, 3, 1445-1474.

Seifert, P. (2012): Mit Sicherheit wächst der Schaden? Überlegungen zum Umgang mit Hochwasser in der räumlichen Planung. Radebeul.

Slovic, P. (1999): Trust, Emotion, Sex, Politics, and Science: Surveying the Risk-Assessment Battlefield. In: Risk Analysis 19, 4, 689-701.

UBA - Umweltbundesamt (Hrsg.) (2015): Monitoringbericht 2015 zur Deutschen Anpassungsstrategie an den Klimawandel. Bericht der Interministeriellen Arbeitsgruppe Anpassungsstrategie der Bundesregierung. www.umweltbundesamt.de/publikationen/ monitoringbericht-2015 (03.06.2015).

UN - United Nations Office for Disaster Risk Reduction (Hrsg.) (2009): Terminology. www.unisdr.org/we/inform/terminology (13.01.2016).

UN - United Nations Office for Disaster Risk Reduction (Hrsg.) (2015): Sendai Framework for Disaster Risk Reduction 20152030. www.unisdr.org/files/43291_sendaiframeworkfordrren.pdf (02.09.2015).

Walker, G.; Tweed, F.; Whittle, R. (2014): A framework for profiling the characteristics of risk governance in natural hazard contexts. In: Natural Hazards and Earth System Sciences 14, 1, 155-164.

WBGU - Wissenschaftlicher Beirat der Bundesregierung Globale Umweltveränderungen (Hrsg.) (1999): Jahresgutachten 1998 „Welt im Wandel - Strategien zur Bewältigung globaler Umweltrisiken". Berlin.

Zehetmair, S. (2011): Konzeptionierung eines Prüfschemas. In: Pohl, J.; Zehetmair, S. (Hrsg.) (2011): Risikomanagement als Handlungsfeld in der Raumplanung. Hannover, 63-70. = ARL Arbeitsmaterial, 357. 